\title{
In vitro experiments on ICOSA6 4D flow MRI measurement for the quantification of velocity and turbulence parameters
}

Hojin Ha, Kyung Jin Park, Petter Dyverfeldt, Tino Ebbers and Dong Hyun Yang

The self-archived postprint version of this journal article is available at Linköping University Institutional Repository (DiVA):

http://urn.kb.se/resolve?urn=urn:nbn:se:liu:diva-169958

N.B.: When citing this work, cite the original publication.

Ha, H., Park, K. J., Dyverfeldt, P., Ebbers, T., Yang, D. H., (2020), In vitro experiments on ICOSA6 4D flow MRI measurement for the quantification of velocity and turbulence parameters, Magnetic Resonance Imaging, 72, 49-6o. https://doi.org/10.1016/j.mri.2020.06.020

Original publication available at:

https://doi.org/10.1016/j.mri.2020.06.020

Copyright: Elsevier

http://www.elsevier.com/

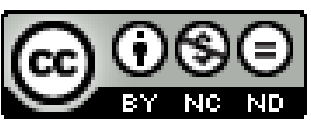


In vitro experiments on ICOSA6 4D flow MRI measurement for the quantification of velocity and turbulence parameters

Hojin $\mathrm{Ha}^{1^{*}, \# \text {, Kyung Jin Park }}{ }^{2,3}$, Petter Dyverfeldt ${ }^{4,5}$, Tino Ebbers ${ }^{4,5}$, Dong Hyun Yang ${ }^{3, \#,}$

${ }^{1}$ Department of Mechanical and Biomedical Engineering, Kangwon National University, Chuncheon, South Korea.

${ }^{2}$ Department of Electrical and Electronic Engineering, Yonsei Univeristy, Seoul, South Korea.

${ }^{3}$ Department of Radiology, University of Ulsan College of Medicine, Asan Medical Center, Seoul, South Korea.

${ }^{4}$ Department of Health, Medicine and Caring Science, Linköping University, Linköping, Sweden.

${ }^{5}$ Center for Medical Image Science and Visualization (CMIV), Linköping University, Linköping, Sweden.

\# These authors are co-corresponding authors for the study

Corresponding Author: Hojin $\mathrm{Ha}^{*}$

Department of Mechanical and Biomedical Engineering,

Kangwon National University,

Chuncheon, Republic of Korea.

Phone: $+82-1092871005$

Fax: $+82-33-259-5548$

E-mail: hojinha@kangwon.ac.kr 


\section{Abstract}

Purpose - To perform comprehensive in vitro experiments using six-directional icosahedral flow encoding (ICOSA6) 4D flow magnetic resonance imaging (MRI) under various scan conditions to analyze the robustness of velocity and turbulence quantification.

Materials and methods - In vitro flow phantoms with steady flow rates of 10 and $20 \mathrm{~L} / \mathrm{min}$ were scanned using both conventional 4D flow MRI and ICOSA6. Experiments focused on comparisons between ICOSA6 and conventional four point (4P) methods, and the effects of contrast agents, velocity encoding range (Venc), and scan direction on velocity and turbulence quantification.

Results - The results demonstrated that 1) ICOSA6 improves the velocity-to-noise ratio (VNR) of velocity estimation by $33 \%$ (on average) and results in similar turbulent kinetic energy (TKE) estimation as the 4P method. 2) Measurements with a contrast agent resulted in more than a 2.5 fold increase in average VNR. However, the improvement of total TKE quantification was not obvious. 3) TKE estimation was less affected by Venc and the scan direction, whereas turbulence production (TP) estimation was largely affected by these measurement conditions. The effects of Venc and scan direction accounted for less than $11.63 \%$ of TKE estimation, but up to $33.89 \%$ of TP estimation.

Conclusion - The ICOSA6 scheme is compatible with conventional 4D flow MRI for velocity and TKE measurement. Contrast agents are effective at increasing VNR, but not signal-to-noise ratio for TKE quantification. The effects of Venc and scan direction influence total TP more than total TKE.

Key words: turbulence; turbulence kinetic energy; Reynolds stress; phase contrast MRI; 4D PC-MRI; 4D Flow MRI 


\section{Abbreviations}

ICOSA6: icosahedral six-directional flow encoding, TKE: turbulent kinetic energy, VNR:

velocity-to-noise ratio, SNR: signal-to-noise ratio, Venc: velocity encoding parameter, TP:

turbulence production, PC-MRI: phase-contrast magnetic resonance imaging, IVVV:

intravoxel velocity variance 


\section{Introduction}

The existence and extent of turbulent blood flow in the cardiovascular system play important roles in the pathogenesis of cardiovascular diseases. High variability in the magnitude and frequency of turbulent flow velocity increases shearing stress on blood components and damages red blood cells [1-3]. Unsteady fluid-dynamic stress caused by turbulence is also responsible for platelet aggregation and thrombus development in disturbed flow regions [4-8]. The proliferation, turnover, and injury of endothelial cell surfaces are sensitively regulated by signal transduction in response to mechanical stimuli [9]. Therefore, turbulence in blood flow is closely related to the initiation, progression, and development of atherosclerosis [9-12].

Although the existence of turbulence in blood flow was first observed several decades ago [13-15], comprehensive descriptions of turbulence in blood flow are still lacking. A few decades ago, catheter-based velocity measurements in humans and animals revealed that turbulence develops not only in the aorta of diseased patients, but also in normal aortic valves [13-15]. Although previous studies have posed various questions regarding the normal range of turbulence in aortic blood flow and whether or not elevated turbulence can be used as an early diagnostic index for vascular diseases, follow-up studies have been rare because of the invasiveness of catheter-based turbulence measurement and a lack of alternatives. However, recently, researchers have non-invasively quantified elevated levels of turbulent flow in the aorta in patients with obstructive cardiovascular diseases, such as aortic valve stenosis and aortic coarctation [16-18].

Time-resolved, three-directional, and three-dimensional phase-contrast magnetic resonance imaging (commonly known as 4D PC-MRI or 4D flow MRI) has been widely used to conduct non-invasive multi-dimensional hemodynamic analysis [19-21]. Recently, 4D flow MRI has not only been used to measure three-dimensional velocity fields in blood flow, but also for estimating the existence and degree of turbulence under in vivo conditions. The 
conventional 4D flow MRI technique measures spatiotemporally averaged velocities within each voxel, meaning the resultant voxel velocity is insensitive to turbulent flow effects. Recently, this technique was extended to estimate the turbulent intensity of small-scale velocity fluctuations in cardiovascular flows $[16,17,22,23]$. A previous study confirmed the feasibility of turbulent kinetic energy (TKE) estimation at clinically practical spatial resolutions [24]. This 4D flow MRI turbulence mapping technique was further generalized with a six-directional icosahedral (ICOSA6) flow-encoding scheme, rather than the conventional four-point (4P) flow-encoding scheme. The generalized technique was successfully applied to measure all components of the Reynolds stress tensor in turbulent flows [25-28]. Recently, the use of a highly undersampled acquisition with locally low-rank image reconstruction showed that turbulence quantification can be performed within ten minutes [29].

Although previous studies using ICOSA6 4D flow MRI have highlighted its clinical potential, fundamental investigations on the measurement of turbulence parameters under various experimental conditions are still lacking. The development of the ICOSA6 scheme and its clinical applications, such as hemodynamic blood damage prediction and irreversible pressure loss estimation, have been described extensively [25-28]. However, questions related to its measurement robustness have not been addressed, including measurement uncertainty/accuracy, comparisons to conventional 4D flow MRI measurement, and possible bias depending on scan direction. In particular, the scan direction may affect the measurement accuracy because it is closely related to the amount of the spatial displacement artifact from high velocity and acceleration in the stenosis [30].

This study aimed to perform comprehensive in vitro experiments using ICOSA6 4D flow MRI under various scan conditions to analyze the robustness of ICOSA6 for turbulence quantifications. This paper presents 1) comparisons between ICOSA6 and conventional 4P methods, 2) an analysis of the effects of contrast agents on velocity and turbulence 
quantification, and 3) an analysis of the effects of velocity encoding (Venc) and scan direction on turbulence quantification.

\section{Methods}

In vitro flow circuit and contraction model

Experimental measurements were conducted using 4D flow MRI on an in vitro flow phantom under various scan conditions to analyze the robustness of ICOSA6 for turbulence quantification. The rectangular flow phantom used for these experiment was a sudden contraction/expansion model with a $75 \%$ reduction in area $(50 \%$ in length, Fig. 1). The upstream size without any constriction is $25 \mathrm{~mm}$. To stabilize the upstream flow in the constriction region, a $0.3 \mathrm{~m}$ straight section was used. Another $0.3 \mathrm{~m}$ straight section was placed downstream from the constriction.

The working fluids were water and a blood analog composed of a 40:60 glycerol/water mixture (by mass). The density and dynamic viscosity of the working fluid were $1053.8 \mathrm{~kg} / \mathrm{m}^{3}$ and $3.72 \times 10^{-3} \mathrm{~kg} \cdot \mathrm{m}^{-1} \cdot \mathrm{s}^{-1}$, respectively. The working fluid was circulated through the flow circuit system at a constant flow rate using a centrifugal pump (EHEIM Universal 3400, Deizisau, Germany). Flow rates of 10 and $20 \mathrm{~L} / \mathrm{min}$ were adopted by monitoring with an electromagnetic flowmeter (VN20, Wintech Process, Korea). Reynolds numbers of the inlet flow at 10 and $20 \mathrm{~L} / \mathrm{min}$ were 1888 and 3777 , respectively. The temperature of the fluid during experimentation was $20^{\circ} \mathrm{C}$. When a contrast agent was used for measurement, $30 \mathrm{~mL}$ of contrast agent $(0.5 \mathrm{mmol} / \mathrm{kg}$, gadofosveset trisodium, VasovistVR, Bayer Schering Pharma AG, Berlin, Germany) was added to $40 \mathrm{~L}$ of the working fluid. 
4D flow MRI measurements were conducted using a clinical 1.5T MRI scanner (1.5T Philips Achieva, Philips Medical Systems, Best, The Netherlands). Three sets of in vitro experiments were conducted: 1) comparison between ICOSA6 and 4P methods, 2) with and without contrast agent in the working fluid, and 3) coronal and axial directional measurements. Coronal measurement sets the frequency encoding direction along the flow direction, whereas axial measurement sets the slice phase encoding along the flow direction. A conventional gradient-echo sequence with asymmetric 4P flow encoding was used for the 4P method which consists of one flow-compensated reference encoding and three directional flow encodings. The ICOSA6 method modifies conventional 4D flow MRI to employ a six-directional icosahedral flow encoding and one flow-compensated reference encoding [28]. Different Venc values ranging from 50 to $450 \mathrm{~cm} / \mathrm{s}$ were used for each experimental condition (see Table 1). The echo time (TE) and temporal resolution ranges were 2.5-3.0 $\mathrm{ms}$ and 3.4-4.4 ms, respectively. The flip angle was $10^{\circ}$. The matrix size range was $128 \times 128 \times(24-70)$ voxels with a $2.0 \mathrm{~mm}$ isotropic voxel size. Partial echo with a factor of 0.725 along the frequency-encoding directions was used to minimize TE. An artificial electrocardiogram with of an interval of $1000 \mathrm{~ms}$ was used to measure and reconstruct multiple phases of turbulent flow. Further details regarding in vitro experiments are summarized in Table 1.

\section{D flow MRI turbulence quantification}

For conventional 4D flow MRI, the MRI signal $\mathrm{S}\left(\mathrm{k}_{\mathrm{v}}\right)$ for a velocity distribution $\mathrm{s}(\mathrm{v})$ is expressed by a Fourier transformation as follows [22, 23]:

$$
S\left(k_{v}\right)=C \int_{-\infty}^{\infty} s(v) e^{-i k_{v} v} d v
$$

where $\mathrm{C}$ is a constant scaling factor influenced by the relaxation parameter, spin density, receiver gain, etc. $\mathrm{k}_{\mathrm{v}}$ represents the level of flow sensitivity, which is related to Venc as $\mathrm{k}_{\mathrm{v}}=\pi$ / Venc. When turbulent flow occurs in the region of interest, the intravoxel velocity variance 
(IVVV) of the turbulent flow along the $i$ direction, denoted $\sigma_{i}^{2}$, can be estimated from the magnitude ratio between the reference signal without velocity encoding $S(0)$ and the signal with velocity encoding along the $i$ direction $\mathrm{S}_{\mathrm{i}}\left(\mathrm{k}_{\mathrm{v}}\right)$ as follows $[22,23]$ :

$$
\sigma_{i}^{2}=\overline{u_{i}^{\prime} u_{i}^{\prime}}=\frac{2}{k_{v}^{2}} \ln \left(\frac{\left|S_{i}(0)\right|}{\left|S_{i}\left(k_{v}\right)\right|}\right)\left(\mathrm{m}^{2} / \mathrm{s}^{2}\right)
$$

Here, $u_{i}^{\prime}$ denotes the fluctuating portion of the velocity and ${ }^{-}$indicates an averaging operation.

The TKE of the flow can be calculated from the IVVV in each direction as follows [31]:

$$
T K E=\frac{1}{2} \rho \sum_{i=1}^{3} \sigma_{i}^{2}=\frac{1}{2} \rho\left(\overline{u_{1}^{\prime} u_{1}^{\prime}}+\overline{u_{2}^{\prime} u_{2}^{\prime}}+\overline{u_{3}^{\prime} u_{3}^{\prime}}\right)\left(\mathrm{J} / \mathrm{m}^{3}\right),
$$

where $\rho$ is the density of the fluid.

When non-orthogonal flow encodings are distributed in a three-dimensional Cartesian space, the obtained velocity and IVVV values can be decomposed into orthogonal components and covariance terms. Therefore, three velocity components (u, v, and w) and the Reynolds stress component tensor $\mathrm{R}_{\mathrm{ij}}$ can be obtained by measuring six non-orthogonal velocity encodings and finding the least-square solutions of the six-directional phase and magnitude data [32]. $\mathrm{R}_{\mathrm{ij}}$ is a six-element symmetric tensor defined as follows:

$$
R=\rho\left[\begin{array}{lll}
\overline{u_{1}^{\prime} u_{1}^{\prime}} & \overline{u_{1}^{\prime} u_{2}^{\prime}} & \overline{u_{1}^{\prime} u_{3}^{\prime}} \\
\overline{u_{2}^{\prime} u_{1}^{\prime}} & \overline{u_{2}^{\prime} u_{2}^{\prime}} & \overline{u_{2}^{\prime} u_{3}^{\prime}} \\
\overline{u_{3}^{\prime} u_{1}^{\prime}} & \overline{u_{3}^{\prime} u_{2}^{\prime}} & \overline{u_{3}^{\prime} u_{3}^{\prime}}
\end{array}\right]
$$

Based on the velocity and Reynolds stress values obtained from the ICOSA6 sequence, turbulent production (TP) can be calculated directly as follows [26]:

$$
T P=-R_{i j} S_{i j},(\mathrm{~J} / \mathrm{kg} \cdot \mathrm{s})
$$

where $S_{i j}$ represents the strain rate tensors of the mean velocity field.

\section{Post-processing of $4 D$ flow MRI data}

All raw data were exported from the scanner using the Pack'n Go tool (Gyrotool LLC, Zurich, Switzerland) and reconstructed using an offline reconstruction tool (ReconFrame, 
Gyrotool LLC, Zurich, Switzerland). Custom MATLAB code was used for the reconstruction of velocity and Reynolds stress values from the icosahedral flow encoding, as described in previous works [25-28]. To extract the velocity of the flow, the acquired data with the highest Venc values $(450,350,300 \mathrm{~m} / \mathrm{s})$ were used. The velocity field obtained without any flow was used to correct the velocity offsets caused by background phase errors [33].

The velocity fluctuation at each voxel was estimated by calculating the standard deviation over all temporal phases and the velocity-to-noise ratio (VNR) was estimated as follows:

$$
\operatorname{VNR}=\frac{\operatorname{mean}(V)}{S D(V)}
$$

where $\mathrm{V}$ is the velocity and SD is the standard deviation.

The signal-to-noise ratio (SNR) of TKE quantification was obtained in a similar manner as follows:

$$
\mathrm{SNR}=\frac{\operatorname{mean}(T K E)}{S D(T K E)}
$$

\section{Results}

Velocity and TKE field measured using ICOSA6 and P4 methods

The ICOSA6 and P4 methods yield qualitatively similar velocity field results for the contraction flow phantom (Fig. 2). The peak velocities measured using ICOSA6 and P4 are $2.78 \pm 0.04 \mathrm{~m} / \mathrm{s}$ and $2.79 \pm 0.06 \mathrm{~m} / \mathrm{s}$, respectively. Compared to the P4 method, ICOSA6 yields less noise in velocity measurements and correspondingly higher VNR levels in both the center and near-wall (the outermost voxels within the channel) regions (Fig. 2b). The increase in VNR when using ICOSA6 compared to $\mathrm{P} 4\left(\mathrm{VNR}_{\mathrm{ICOSA}_{6}} / \mathrm{VNR}_{\mathrm{P} 4}\right)$ is $1.33 \pm 0.49$ ( $\operatorname{minimum}=0.21$, maximum $=11.93$ ). Therefore, ICOSA6 provides a 33\% average VNR increase over the entire flow domain. Bland-Altman analysis revealed that the slope of linear regression for the velocity 
measurement was 1.01 with $\mathrm{r}^{2}=0.98$ and that the mean bias with a $95 \%$ limit of agreement was $0.01 \pm 0.14 \mathrm{~m} / \mathrm{s}$ (Figure S1 in the Supporting Information).

The ICOSA6 and P4 methods yield qualitatively similar TKE fields in the post-stenotic region (Fig. 3). The slopes of linear regression for TKE estimation are 0.89, 0.89, 0.95, and 1.03 at Venc values of $200,150,100$, and $50 \mathrm{~cm} / \mathrm{s}$, respectively. The mean biases with a $95 \%$ limit of agreement are $-6.62 \pm 112.48,-5.88 \pm 88.37,-3.45 \pm 56.52$, and $-0.54 \pm 19.77 \mathrm{~mJ}$ at Venc values of 200, 150, 100, and $50 \mathrm{~cm} / \mathrm{s}$, respectively (Figure S2 in the Supporting Information). Both methods yield similar total TKE values between 11.54 and $12.37 \mathrm{~mJ}$ with Venc values in the range of $100-200 \mathrm{~cm} / \mathrm{s}$ (Table 2). A Venc of $50 \mathrm{~cm} / \mathrm{s}$ resulted in an underestimation of the TKE, so the ICOSA6 and P4 methods yield small total TKE values of $7.98 \pm 0.11$ and $7.93 \pm 0.10 \mathrm{~mJ}$, respectively (Fig. 3 and Table 1). Although ICOSA6 yields approximately $5.90 \%$ to $16.94 \%$ less TKE noise upstream of the contraction (Table 2), the TKE fluctuations and corresponding SNRs in the peak TKE region reveal that there is no significant difference in terms of TKE quantification between ICOSA6 and P4 (Fig. 4). However, the SNR of TKE quantification does vary with the choice of Venc for measurement (Fig. 4). The voxelwise noise level and SNR of the velocity and TKE over the entire flow domain are provided in Figure S3 and S4 in the Supporting Information.

\section{Velocities and Reynolds stress fields measured with and without a contrast agent}

Introducing a contrast agent improves velocity field visualization over the contraction flow phantom (Fig. 5). The peak velocities measured with and without the contrast agent are $2.91 \pm 0.05 \mathrm{~m} / \mathrm{s}$ and $2.86 \pm 0.14 \mathrm{~m} / \mathrm{s}$, respectively. Measurement with the contrast agent results in less velocity noise and correspondingly enhanced VNR levels in both the center and nearwall regions (Fig. 5b). In VNR enhancement measured with the contrast agent ( $\mathrm{VNR}_{\text {Contrast }} /$ 
$\mathrm{VNR}_{\text {Non-contrast }}$ ) is $2.56 \pm 1.06$ (minimum $=0.46$, maximum $\left.=10.88\right)$. In other words, the contrast agent yields more than a 2.5 fold increase in average VNR over the entire flow domain.

Measurements with and without a contrast agent result in qualitatively similar TKE fields in the post-stenotic region (Fig. 6). Both methods yield total TKE values between 12.16 and $13.69 \mathrm{~mJ}$ with Venc values in the range of 100-200 cm/s (Table 3). Again, a Venc of 50 $\mathrm{cm} / \mathrm{s}$ resulted in an underestimate of the TKE, so ICOSA6 and P4 measurements resulted in small total TKE values of $10.07 \pm 0.09$ and $9.72 \pm 0.08 \mathrm{~mJ}$, respectively (Fig. 6 and Table 3). While measurement with the contrast agent results in approximately $17.75 \%$ to $53.28 \%$ less TKE noise upstream of the contraction (Table 3), the TKE fluctuations and corresponding SNRs in the peak TKE regions demonstrate that there is no significant improvement in TKE quantification with the contrast agent. However, the SNR of TKE quantification does vary with the choice of Venc for measurement (Fig. 7).

TP estimation with the contrast agent results in a lower noise level in the post-stenotic region (Fig. 8). The total TP values with the contrast agent are $549.90 \pm 18.52 \mathrm{~mW}, 660.42 \pm$ $21.16 \mathrm{~mW}, 616.99 \pm 14.96 \mathrm{~mW}$, and $72.26 \pm 5.55 \mathrm{~mW}$ for Venc values of $200,150,100$, and $50 \mathrm{~cm} / \mathrm{s}$, respectively (Table 4). The total TP values without the contrast agent are $514.59 \pm$ $33.75 \mathrm{~mW}, 625.18 \pm 22.34 \mathrm{~mW}, 564.42 \pm 21.23 \mathrm{~mW}$, and $59.16 \pm 7.02 \mathrm{~mW}$ for Venc values of $200,150,100$, and $50 \mathrm{~cm} / \mathrm{s}$, respectively. The mean differences between these measurements are $6.42 \%, 5.34 \%, 8.07 \%$, and $18.13 \%$ for Venc values of $200,150,100$, and $50 \mathrm{~cm} / \mathrm{s}$, respectively (Table 4). Total TP variations of $16.73 \%$ and $17.69 \%$ were observed for Venc values of 100 and $200 \mathrm{~cm} / \mathrm{s}$, respectively. Therefore, the choice of Venc has a greater effect than the use of a contrast agent for TP estimation.

It was determined that Venc-dependent changes in the total TP can be largely attributed to changes in the Reynolds stress component depending on the choice of Venc (Fig. 9). The total $\mathrm{TP}_{\mathrm{xz}}$ associated with $\mathrm{R}_{\mathrm{xz}}$ increases as the Venc increases from 100 to $200 \mathrm{~cm} / \mathrm{s}$, while the 
total $\mathrm{TP}_{\mathrm{yz}}$ associated with $\mathrm{R}_{\mathrm{yz}}$ decreases as the Venc increases from 100 to $200 \mathrm{~cm} / \mathrm{s}$ (Table 5). These phenomena result in two-directional Reynolds stress $\left(\mathrm{R}_{\mathrm{xz}}\right.$ and $\left.\mathrm{R}_{\mathrm{yz}}\right)$ components that vary with the choice of Venc. The regions with high $\mathrm{R}_{\mathrm{xz}}$ values around the contraction area are suppressed at low Venc values (marked as * and ** in Fig. 9). In contrast, $\mathrm{R}_{\mathrm{yz}}$ decreases at high Venc values (marked as $* * *$ in Fig. 9).

\section{Comparison of ICOSA6 measurements with axial and coronal scan directions}

Coronal and axial methods result in qualitatively similar TKE fields in the post-stenotic region (Fig. 10). Both methods result in total TKE values between 12.65 and $13.81 \mathrm{~mJ}$ at a flow rate of $20 \mathrm{~L} / \mathrm{min}$ with Venc values of 100 to $200 \mathrm{~cm} / \mathrm{s}$ and corresponding mean differences of $0.65 \%$ to $4.60 \%$. Both methods yield total TKE values between 3.60 and $4.17 \mathrm{~mJ}$ at a flow rate of $10 \mathrm{~L} / \mathrm{min}$ with Venc values of 100 to $200 \mathrm{~cm} / \mathrm{s}$ and corresponding mean differences of $10.89 \%$ to $11.51 \%$ (Table 6 ). Venc values of 75 and $50 \mathrm{~cm} / \mathrm{s}$ were found to be too small to estimate the TKE, so both coronal and axial directional measurements tend to underestimate the total TKE (Table 6.)

TP estimation is also influenced by the choice of measurement direction and Venc (Fig. 11). It was determined that the total $\mathrm{TP}$ varies by $22.18 \%$ and $24.14 \%$ with measurement direction at Venc values of 100 to $200 \mathrm{~cm} / \mathrm{s}$ and flow rates of 20 and $10 \mathrm{~L} / \mathrm{min}$, respectively (Table 7). The coronal directional measurements reveal that total TP varies by $16.51 \%$ and $10.99 \%$ depending on the choice of Venc between 100 and $200 \mathrm{~cm} / \mathrm{s}$ with flow rates of 20 and $10 \mathrm{~L} / \mathrm{min}$, respectively (Table 7). The axial directional measurements reveal that total TP varies by $32.76 \%$ and $33.89 \%$ depending on the choice of Venc between 100 and $200 \mathrm{~cm} / \mathrm{s}$ with flow rates of 10 and $20 \mathrm{~L} / \mathrm{min}$, respectively (Table 7).

\section{Discussion}


In this study, we aimed to perform comprehensive in vitro experiments using ICOSA6 4D flow MRI under various scan conditions to analyze the robustness of ICOSA6 for turbulence quantification. Our major findings can be summarized as follows. 1) ICOSA6 improves the average VNR of velocity estimation by $33 \%$ and results in similar TKE estimation compared to the 4P method. 2) Measurement with a contrast agent results in more than a 2.5 fold increase in average VNR, but the improvement of total TKE quantification is not obvious. 3) TKE estimation is barely affected by Venc and scan direction, whereas TP estimation is significantly affected by measurement conditions. The effects of Venc and scan direction are less than $11.51 \%$ in terms of TKE estimation, but as high as $33.89 \%$ in terms of TP estimation.

This study demonstrates that TKE estimations using ICOSA6 and 4P method are linearly correlated. Although previous studies on ICOSA6 have shown promising potential for Reynolds stress and TP quantifications [27, 28], agreements of the flow parameters measured by ICOSA6 and conventional 4P methods have not been confirmed. This study demonstrated that ICOSA6 provides comparable TKE quantification with a superior VNR for velocity measurement compared to the conventional $4 \mathrm{P}$ method. The increase in VNR largely stems from the compensation of enhanced flow encoding and scan time provided by ICOSA6 [34].

A superior VNR of the ICOSA6 compared to the conventional 4P method may not guarantee better quantification of the flow rate through the stenotic channel. While the maximum difference of the peak velocity through the stenosis was less than around $15 \%$, the flow rate estimation at the post-stenosis region was largely deteriorated in both sequences (Table S3 and Figure S5). While the upstream flow rate was measured to around $20 \mathrm{~L} / \mathrm{min}$, both ICOSA6 and P4 methods underestimated the flow rate at the stenosis apex and overestimated at the post-stenosis. This result suggest that the flow rate estimation should be estimated prior to the contraction, if possible. 
This study also shows that the increase of SNR by a contrast-agent does not effectively improve the uncertainty of the TKE quantification. A previous study on the effects of contrast agents on 4D flow MRI reported that the use of a contrast agent approximately doubles the SNR of measurements [35]. Our study agrees with these previous results. Specifically, the baseline noise levels of velocity measurements were reduced by approximately half when using a contrast agent (Fig. 5). However, this did not improve the qualitative visualization of the TKE field or the SNR of TKE quantification (Fig. 6 and Table 3). This finding can be attributed to significant TKE fluctuation around the turbulent-flow region, regardless of the use of a contrast agent (Fig. S3 in the Supporting Information). Although the k-space for 4D flow MRI is iteratively acquired over hundreds to thousands of TRs, most low-frequency signals stem from the few lines of k-space around the center. Therefore, measurement inevitably yields a statistically non-converged TKE field with scan-to-scan variance. This scan-to-scan TKE fluctuation is greater than the background noise level. Therefore, the benefit of contrast agent in terms of TKE quantification only appears at locations with low-turbulence regions, such as upstream of the contraction.

The present results indicate that SNR may not be the primary parameter determining the uncertainty of turbulence quantifications. Previous studies widely investigated the effect of SNR on the turbulence quantifications. Previously, Binter et al. investigated the limits of MRI turbulence quantifications with respect to the spatial resolution, SNR, and intravoxel velocity distribution, and suggested a SNR of 20 to 30 is required in general [24]. Ha and Park also simulated the uncertainty of the turbulence quantifications at various SNRs and showed that SNR over 20 is needed for ICOSA6 measurement [36]. However, both studies assume that MRI encodes a sufficient number of samples for obtaining turbulence parameters because the measurements are built up by samples taken over both space and time during the repetitive kspace acquisitions [22]. Yet, this study experimentally demonstrated that the current acquisition 
still yields a statistically non-converged TKE field with a scan-to-scan variance. This indicates that the scan-to-scan variance due to insufficient sampling for the turbulence quantification should be considered even though sufficient SNR is achieved for the measurement.

The choice of Venc affects TP quantification more than TKE quantification. Practical choices for Venc in terms of stenotic aortic flow have been reported to be approximately 100 to $200 \mathrm{~cm} / \mathrm{s}[16,17]$. The maximum differences in total TKE and TP over the practical range are $11.51 \%$ and $33.89 \%$, respectively (Table 3 and 7 ). The differences in $\mathrm{TP}$ are largely attributed to Venc-dependent changes in off-diagonal Reynolds stress ( $\mathrm{R}_{\mathrm{xz}}$ and $\mathrm{R}_{\mathrm{yz}}$, Fig. 9). A previous study demonstrated that TKE measurement with a low Venc value that results in a turbulence-related signal magnitude much less than $60 \%$ of the original signal magnitude can lead to the underestimation of turbulence parameters based on the nonzero Rician distribution of noise [22]. The results presented in this paper agree with those of previous studies showing $\mathrm{R}_{\mathrm{xz}}$ underestimation with low-Venc measurements. $\mathrm{R}_{\mathrm{xz}}$, rather than $\mathrm{R}_{\mathrm{yz}}$, was underestimated in this study because the z-directional moments of the ICOSA6 scheme are greater for $\mathrm{R}_{\mathrm{xz}}$ measurement than they are for $\mathrm{R}_{\mathrm{yz}}$ measurement [28]. In contrast, $\mathrm{R}_{\mathrm{yz}}$ was underestimated when using high-Venc measurements. The exact mechanism of the underestimation of turbulence parameters with high-Venc measurements is not fully understood, so it should be investigated further.

The choice of measurement direction also affects TP quantification more than TKE quantification. The maximum differences in the total TKE and TP values over the practical range (100-200 cm/s) are $11.63 \%$ and $24.12 \%$, respectively (Table 6 and 7 ). It is possible that the effect of the scan direction on TP is the same as the previously described Venc effect. Because the choice of the measurement direction (coronal or axial) determines all six directional flow-encoding moments relative to the flow direction, the resultant effect can be incorporated with the Venc effect on TP quantification. 
Although turbulence quantification using ICOSA6 results in a significant amount of negative TP, the robust treatment of negative TP in a turbulent flow has not yet been discussed. Unlike to positive TP, which indicates energy transfer from the mean flow to the fluctuating velocity, negative $\mathrm{TP}$ is a rarely observable phenomenon because it indicates energy backscattering from the fluctuating velocity to the mean flow $[26,37,38]$. Therefore, it is likely that most of the negative TP values associated with stenotic flow stem from incorrect measurements. To minimize measurement error, previous studies have employed the multiVenc approach to obtain the best estimates possible and filter negative TP for quantification $[26,27]$. A recent study used another approach and filtered only the negative diagonal components of Reynolds stress since the diagonal terms cannot be negative by definition [39]. However, both approaches still maintain the Venc and directional effects on TP quantification (Table S1 and S2). Therefore, the robust treatment of negative TP in turbulent flows should be investigated further by comparing observed values to ground-truth data, such as data derived from a direct numerical simulation.

The present study suggests that the Venc range between $100 \mathrm{~cm} / \mathrm{s}$ to $200 \mathrm{~cm} / \mathrm{s}$ is feasible for ICOSA6 turbulence quantification, but that the use of contrast-agents is not effective for reducing the turbulence measurement uncertainty. Although the spatial resolution and temporal resolution have not been included for the present study, they should also be considered as they also can affect the turbulence quantifications. The spatial resolution has been known to affect the peak TKE value but does not affect total the TKE for spatial resolutions between $1 \mathrm{~mm}$ and $2 \mathrm{~mm}$ [40]. Furthermore, using simulations of the MRI acquisition, it was shown that the TP parameter can be accurately quantified within a range of practical spatial resolutions between $1 \mathrm{~mm}$ to $3 \mathrm{~mm}$, preferably less than $2.4 \mathrm{~mm}$ [26]. As the low temporal resolution underestimates the peak flow velocity, the turbulence parameters can be also affected by the temporal resolution $[41,42]$. While the higher temporal resolution would result in better accuracy, the 
temporal resolution around $40 \mathrm{~ms}$ is currently suggested due to the scan time limitation. The inherently longer acquisition time of temporally resolved acquisitions might have some positive effect on the scan-to-scan variance, as there is more time between adjacent k-space lines. Larger reduction of these effects might however be obtained by k-space sampling patterns which included repeated sampling of the central part of k-space.

As the uncertainty and accuracy of the ICOSA6 measurement are assured, more clinical applications of the turbulent quantification can be explored. The TKE in the blood flow has been a hemodynamic index quantifying pathologic turbulent blood flow due to the diseased vessel and heart valve [29]. It was also proposed as an alternative index for predicting the irreversible pressure drop across a stenosis [16]. However, the TKE based pressure estimation is dependent on both the vessel geometry and stenosis severity which limits its potential [43]. Therefore, TP, which is the amount of energy drawn from the mean flow to produce turbulence, has been proposed to estimate the irreversible pressure drop [26]. Since the TP estimation requires all components of the Reynolds stress tensor, the ICOSA6 was required instead of the conventional 4P. In addition, the quantification of the Reynolds stress has been proposed to predict hemodynamic blood damage due to the turbulent stress [44].

However, the longer acquisition time of ICOSA6 turbulence quantification compared to the $4 \mathrm{P}$ method has been an inherent drawback. Since ICOSA6 employs a total of seven flow encodings rather than four directional conventional flow encodings, ICOSA6 requires a $75 \%$ longer scan time. Recently various acceleration techniques such as compressed sensing and local low-rank have been developed and successfully applied to reduce the scan time $[45,46]$. Walheim et al. presented highly undersampled 5D Flow MRI acquisition with locally low-rank image reconstruction and showed that turbulence quantification can be performed within ten minutes [29]. We speculate that the scan time of the turbulence quantification will become trivial as the acceleration techniques are further developed. 
The use of a steady flow reduced the complexity of the study, facilitating the examination of other desired parameters, such as the effects of Venc and measurement direction. However, the main limitation of this study is that the effect of pulsatile flow was not examined. While the higher temporal resolution would result in the better accuracy, the temporal resolution around $40 \mathrm{~ms}$ is currently suggested for 4D Flow MRI due to the scan time limitation [42]. Since the turbulence quantification with insufficient temporal resolution is expected to underestimate the turbulence parameters due to the temporal averaging effect, MRI turbulence quantification for in-vivo pulsatile blood flow should be carried out with a similar temporal resolution so that the temporal resolution effect can be minimized. The results presented in this paper were all obtained using a static flow phantom. Therefore, the effects of motion and gating issues during the scanning of in vivo subjects must still be been investigated.

In addition, while the present study analyzes the measurement uncertainty mostly from the comprehensive experiments, accuracy of the measurement is still unknown mostly due to the lack of ground truth turbulence data. While previously laser Doppler velocimetry and particle image velocimetry have been used to validate the MRI turbulence quantifications [47, 48], those experimental measurements usually provide velocity information within the limited region so that the entire flow field cannot be compared. While the measurement can be compared with computational fluid dynamics (CFD) simulations, most of CFD simulation employs their own turbulence models such as Reynolds-averaged Navier-Stokes or large-eddy simulation turbulence models. The accuracy of these turbulence models should be also questioned until experimentally confirmed [49]. Therefore, further validation studies are desired to compare the measurement with direct numerical simulation because it provides highfidelity solution of turbulent flow despite of extreme computational cost and the challenges of obtaining the same conditions for the simulations and measurements [50]. 


\section{Conclusion}

The ICOSA6 scheme is compatible with conventional 4D flow MRI for velocity and TKE measurement. Contrast agents are effective for increasing VNR, but not the SNR for TKE quantification. The choice of Venc and scan direction has a greater influence on total TP than on total TKE.

\section{Data availability}

All measurement data can be accessed from https://osf.io/g3zb8/. All data are directly from the scanner and can be reconstructed with any reconstruction software.

\section{Acknowledgments}

None.

\section{Funding}

This research was supported by the Basic Science Research Program through the National Research Foundation of Korea, which is funded by the Ministry of Education (2018R1D1A1A02043249). This research was also supported by the Basic Science Research Program, through the National Research Foundation of Korea (NRF), funded by the Ministry of Science, ICT \& Future Planning (NRF-2020R1A2C2003843).

\section{Disclosures}

The authors declare no competing financial interests.

\section{References}

[1] Lu P, Lai H, Liu J. A reevaluation and discussion on the threshold limit for hemolysis in a turbulent shear flow. Journal of biomechanics 2001;34(10):1361-4.

[2] Sallam AM, Hwang N. Human red blood cell hemolysis in a turbulent shear flow: contribution of Reynolds shear stresses. Biorheology 1983;21(6):783-97.

[3] Yen J-H, Chen S-F, Chern M-K, Lu P-C. The effect of turbulent viscous shear stress 
on red blood cell hemolysis. Journal of Artificial Organs 2014;17(2):178-85.

[4] Mustard J, Murphy E, Rowsell H, Downie H. Factors influencing thrombus formation in vivo. The American journal of medicine 1962;33(5):621-47.

[5] Smith RL, Blick EF, Coalson J, Stein PD. Thrombus production by turbulence. Journal of applied physiology 1972;32(2):261-4.

[6] Stein P, Walburn F, Sabbah H. Turbulent stresses in the region of aortic and pulmonary valves. Journal of biomechanical engineering 1982;104(3):238-44.

[7] Stein PD, Sabbah HN, Pitha JV. Continuing disease process of calcific aortic stenosis: role of microthrombi and turbulent flow. The American journal of cardiology 1977;39(2):15963.

[8] Yoganathan AP, Woo Y-R, Sung H-W. Turbulent shear stress measurements in the vicinity of aortic heart valve prostheses. Journal of biomechanics 1986;19(6):433-42.

[9] Davies PF, Remuzzi A, Gordon EJ, Dewey CF, Gimbrone MA. Turbulent fluid shear stress induces vascular endothelial cell turnover in vitro. Proceedings of the National Academy of Sciences 1986;83(7):2114-7.

[10] Davies PF. Hemodynamic shear stress and the endothelium in cardiovascular pathophysiology. Nature clinical practice Cardiovascular medicine 2009;6(1):16-26.

[11] Mehta V, Tzima E. Cardiovascular disease: A turbulent path to plaque formation. Nature 2016;540(7634):531-2.

[12] Wang L, Luo J-Y, Li B, Tian XY, Chen L-J, Huang Y, et al. Integrin-YAP/TAZ-JNK cascade mediates atheroprotective effect of unidirectional shear flow. Nature 2016.

[13] Stein PD, Sabbah HN. Turbulent blood flow in the ascending aorta of humans with normal and diseased aortic valves. Circulation research 1976;39(1):58-65.

[14] Yamaguchi T, Kikkawa S, Yoshikawa T, Tanishita K, Sugawara M. Measurement of turbulence intensity in the center of the canine ascending aorta with a hot-film anemometer. Journal of biomechanical engineering 1983;105(2):177-87.

[15] Hanai S, Yamaguchi T, Kikkawa S. Turbulence in the canine ascending aorta and the blood pressure. Biorheology 1991;28(1-2):107-16.

[16] Dyverfeldt P, Hope MD, Tseng EE, Saloner D. Magnetic resonance measurement of turbulent kinetic energy for the estimation of irreversible pressure loss in aortic stenosis.

JACC: Cardiovascular Imaging 2013;6(1):64-71.

[17] Dyverfeldt P, Kvitting JPE, Sigfridsson A, Engvall J, Bolger AF, Ebbers T.

Assessment of fluctuating velocities in disturbed cardiovascular blood flow: in vivo feasibility of generalized phase-contrast MRI. Journal of Magnetic Resonance Imaging: An Official Journal of the International Society for Magnetic Resonance in Medicine 2008;28(3):655-63.

[18] Ha H, Ziegler M, Welander M, Bjarnegård N, Carlhäll C-J, Lindenberger M, et al. Age-related vascular changes affect turbulence in aortic blood flow. Frontiers in physiology 2018;9:36.

[19] Caruthers SD, Lin SJ, Brown P, Watkins MP, Williams TA, Lehr KA, et al. Practical value of cardiac magnetic resonance imaging for clinical quantification of aortic valve stenosis. Circulation 2003;108(18):2236-43.

[20] Donati F, Myerson S, Bissell MM, Smith NP, Neubauer S, Monaghan MJ, et al. Beyond Bernoulli. Circulation: Cardiovascular Imaging 2017;10(1):e005207.

[21] Falahatpisheh A, Rickers C, Gabbert D, Heng EL, Stalder A, Kramer HH, et al. Simplified Bernoulli's method significantly underestimates pulmonary transvalvular pressure drop. Journal of Magnetic Resonance Imaging 2016;43(6):1313-9.

[22] Dyverfeldt P, Gårdhagen R, Sigfridsson A, Karlsson M, Ebbers T. On MRI turbulence quantification. Magnetic resonance imaging 2009;27(7):913-22.

[23] Dyverfeldt P, Sigfridsson A, Kvitting JPE, Ebbers T. Quantification of intravoxel velocity standard deviation and turbulence intensity by generalizing phase-contrast MRI. Magnetic Resonance in Medicine 2006;56(4):850-8.

[24] Binter C, Gülan U, Holzner M, Kozerke SJMrim. On the accuracy of viscous and turbulent loss quantification in stenotic aortic flow using phase-contrast MRI. 2016;76(1):1916.

[25] Ha H, Lantz J, Haraldsson H, Casas B, Ziegler M, Karlsson M, et al. Assessment of 
turbulent viscous stress using ICOSA 4D Flow MRI for prediction of hemodynamic blood damage. Scientific reports 2016;6(1):1-14.

[26] Ha H, Lantz J, Ziegler M, Casas B, Karlsson M, Dyverfeldt P, et al. Estimating the irreversible pressure drop across a stenosis by quantifying turbulence production using 4D Flow MRI. Scientific reports 2017;7:46618.

[27] Ha H, Kvitting JP, Dyverfeldt P, Ebbers T. Validation of pressure drop assessment using 4D flow MRI-based turbulence production in various shapes of aortic stenoses. Magnetic resonance in medicine 2019;81(2):893-906.

[28] Haraldsson H, Kefayati S, Ahn S, Dyverfeldt P, Lantz J, Karlsson M, et al. Assessment of Reynolds stress components and turbulent pressure loss using 4D flow MRI with extended motion encoding. Magnetic resonance in medicine 2018;79(4):1962-71.

[29] Walheim J, Dillinger H, Gotschy A, Kozerke SJSR. 5D Flow Tensor MRI to Efficiently Map Reynolds Stresses of Aortic Blood Flow In-Vivo. 2019;9(1):1-12.

[30] Petersson S, Dyverfeldt P, Sigfridsson A, Lantz J, Carlhäll CJ, Ebbers TJMrim. Quantification of turbulence and velocity in stenotic flow using spiral three-dimensional phase-contrast MRI. 2016;75(3):1249-55.

[31] Pope SB. Turbulent flows. IOP Publishing; 2001.

[32] Haraldsson H, Kefayati S, Ahn S, Dyverfeldt P, Lantz J, Karlsson M, et al. Assessment of Reynolds stress components and turbulent pressure loss using 4D flow MRI with extended motion encoding. Magnetic Resonance in Medicine 2017.

[33] Petersson S, Dyverfeldt P, Sigfridsson A, Lantz J, Carlhäll CJ, Ebbers T. Quantification of turbulence and velocity in stenotic flow using spiral three-dimensional phase-contrast MRI. Magnetic resonance in medicine 2016;75(3):1249-55.

[34] Zwart NR, Pipe JG. Multidirectional high-moment encoding in phase contrast MRI. Magnetic resonance in medicine 2013;69(6):1553-63.

[35] Bock J, Frydrychowicz A, Stalder AF, Bley TA, Burkhardt H, Hennig J, et al. 4D phase contrast MRI at $3 \mathrm{~T}$ : Effect of standard and blood-pool contrast agents on SNR, PCMRA, and blood flow visualization. 2010;63(2):330-8.

[36] Ha H, Park HJJoV. Comparison of turbulent flow measurement schemes for 4D flow MRI. 2019;22(3):541-53.

[37] Liberzon A, Lüthi B, Guala M, Kinzelbach W, Tsinober A. Experimental study of the structure of flow regions with negative turbulent kinetic energy production in confined threedimensional shear flows with and without buoyancy. Physics of Fluids 2005;17(9):095110.

[38] Beguier C, Giralt F, Fulachier L, Keffer J. Negative production in turbulent shear flows. Structure and Mechanisms of Turbulence II. Springer; 1978, p. 22-35.

[39] Marlevi D, Ha H, Dillon-Murphy D, Fernandes JF, Fovargue D, Colarieti-Tosti M, et al. Non-invasive estimation of relative pressure in turbulent flow using virtual work-energy. Medical Image Analysis 2020;60:101627.

[40] Casas B, Lantz J, Dyverfeldt P, Ebbers TJMrim. 4D flow MRI-Based pressure loss estimation in stenotic flows: Evaluation using numerical simulations. 2016;75(4):1808-21.

[41] Carlsson M, Töger J, Kanski M, Bloch KM, Ståhlberg F, Heiberg E, et al. Quantification and visualization of cardiovascular 4D velocity mapping accelerated with parallel imaging or kt BLAST: head to head comparison and validation at $1.5 \mathrm{~T}$ and $3 \mathrm{~T}$. 2011;13(1):55.

[42] Dyverfeldt P, Bissell M, Barker AJ, Bolger AF, Carlhäll C-J, Ebbers T, et al. 4D flow cardiovascular magnetic resonance consensus statement. 2015;17(1):72.

[43] Casas B, Lantz J, Dyverfeldt P, Ebbers T. 4D flow MRI-Based pressure loss estimation in stenotic flows: Evaluation using numerical simulations. Magnetic resonance in medicine 2016;75:1808-21.

[44] Ha H, Lantz J, Haraldsson H, Casas B, Ziegler M, Karlsson M, et al. Assessment of turbulent viscous stress using ICOSA 4D Flow MRI for prediction of hemodynamic blood damage. 2016;6(1):1-14.

[45] Ma LE, Markl M, Chow K, Huh H, Forman C, Vali A, et al. Aortic 4D flow MRI in 2 minutes using compressed sensing, respiratory controlled adaptive k-space reordering, and inline reconstruction. 2019;81(6):3675-90. 
[46] Zhang T, Pauly JM, Levesque IRJMrim. Accelerating parameter mapping with a locally low rank constraint. 2015;73(2):655-61.

[47] Dyverfeldt P, Sigfridsson A, Kvitting JPE, Ebbers TJMRiMAOJotISfMRiM. Quantification of intravoxel velocity standard deviation and turbulence intensity by generalizing phase-contrast MRI. 2006;56(4):850-8.

[48] Ha H, Kim GB, Kweon J, Huh HK, Lee SJ, Koo HJ, et al. Turbulent kinetic energy measurement using phase contrast MRI for estimating the post-stenotic pressure drop: in vitro validation and clinical application. 2016;11(3).

[49] Miyazaki S, Itatani K, Furusawa T, Nishino T, Sugiyama M, Takehara Y, et al. Validation of numerical simulation methods in aortic arch using 4D Flow MRI. 2017;32(8):1032-44.

[50] Lui M, Martino S, Salerno M, Quadrio MJJobe. On the turbulence modeling of blood flow in a stenotic vessel. 2020;142(1). 


\section{Figure Legends}

Figure 1. Experimental setup. (a) schematic of the flow circuit, (b) geometry of the rectangular sudden contraction flow model.

Figure 2. Comparison of ICOSA6 and P4 velocity measurements. (a) velocity field, (b) velocity and velocity-to-noise ratio (VNR) along the centerline and near-wall region. Note that the near-wall region indicates the outermost voxel of the phantom. $\mathrm{X}=0$ at the contraction region and $\mathrm{D}$ is the upstream size of $25 \mathrm{~mm}$.

Figure 3. Comparison TKE field measured with ICOSA6 and P4 sequences. (b) Single-scan TKE field, (b) average TKE field of 30 phases.

Figure 4. Comparison TKE field along the centerline measured with ICOSA6 and P4 sequences. Data points show the mean $\pm \mathrm{SD} . \mathrm{X}=0$ at the contraction region and $\mathrm{D}$ is the upstream size of $25 \mathrm{~mm}$.

Figure 5. Comparison of velocity measurements with and without contrast agent. (a) velocity field, (b) velocity and velocity-to-noise ratio (VNR) along the centerline and near-wall region. Note that the near-wall region indicates the outermost voxel of the phantom. $\mathrm{X}=0$ at the contraction region and $\mathrm{D}$ is the upstream size of $25 \mathrm{~mm}$.

Figure 6. Comparison TKE field measured with and without contrast agent. (b) Single-scan TKE field, (b) average TKE field of 30 phases.

Figure 7. Comparison TKE field along the centerline measured with and without contrast agent. Data points show the mean $\pm \mathrm{SD}$. $\mathrm{X}=0$ at the contraction region and $\mathrm{D}$ is the upstream size of $25 \mathrm{~mm}$.

Figure 8. Comparison turbulence production field measured with and without contrast agent. (b) Single-scan TKE field, (b) average TKE field of 30 phases.

Figure 9. Comparison two directional Reynolds stress $\left(\mathrm{R}_{\mathrm{xy}}\right.$ and $\left.\mathrm{R}_{\mathrm{yz}}\right)$ at different velocity encoding (VENC) parameters. (b) Instantaneous TKE field, (b) average TKE field of 30 phases.

Figure 10. TKE field measured with coronal and axial directional measurements

Figure 11. Turbulence production field measured with coronal and axial directional measurement 


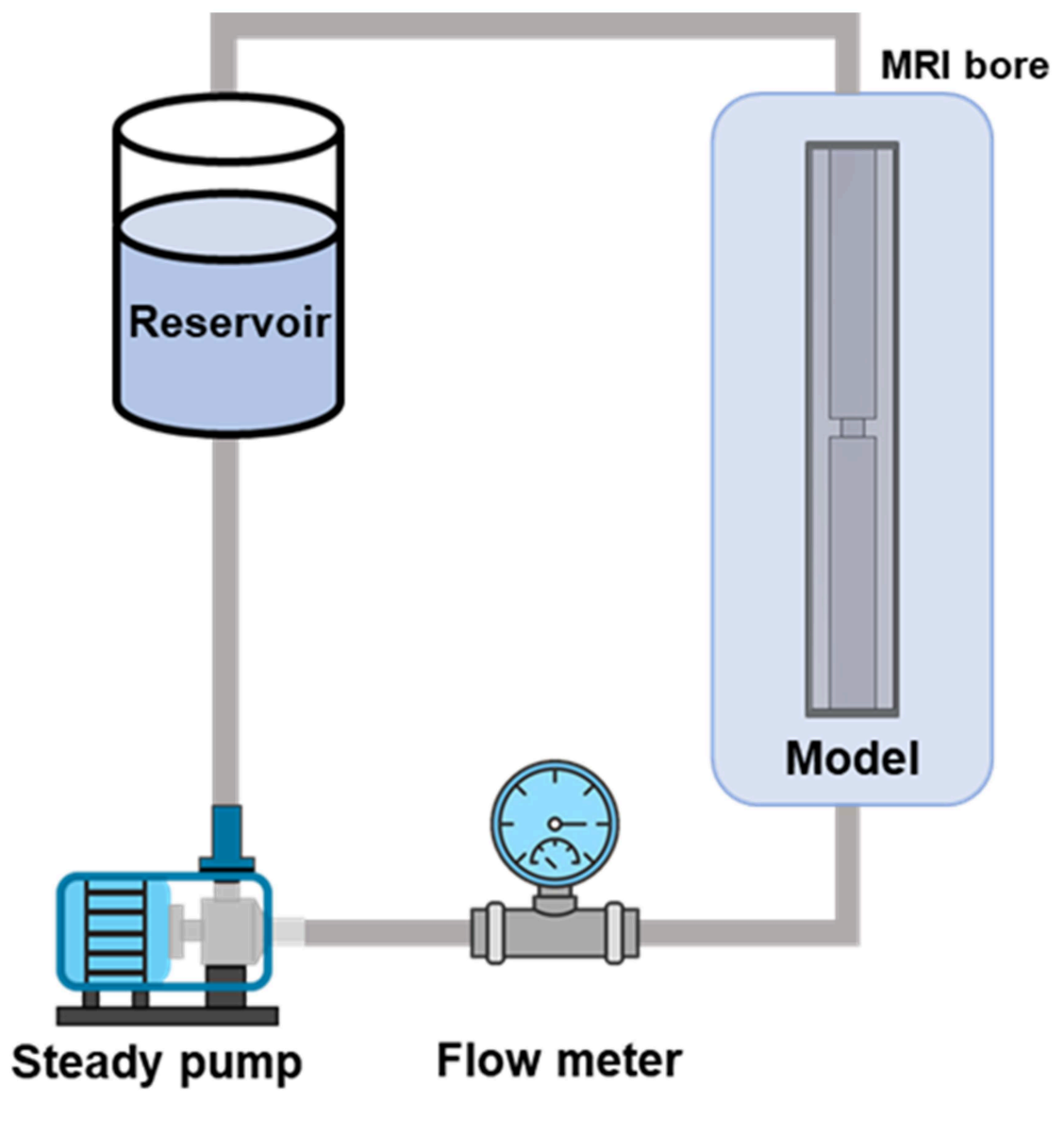

\section{Flow direction}

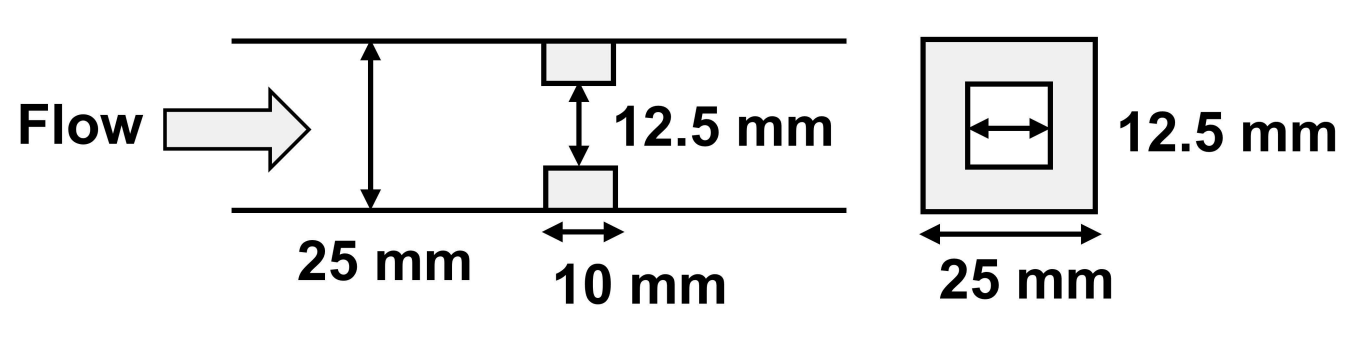


(b)
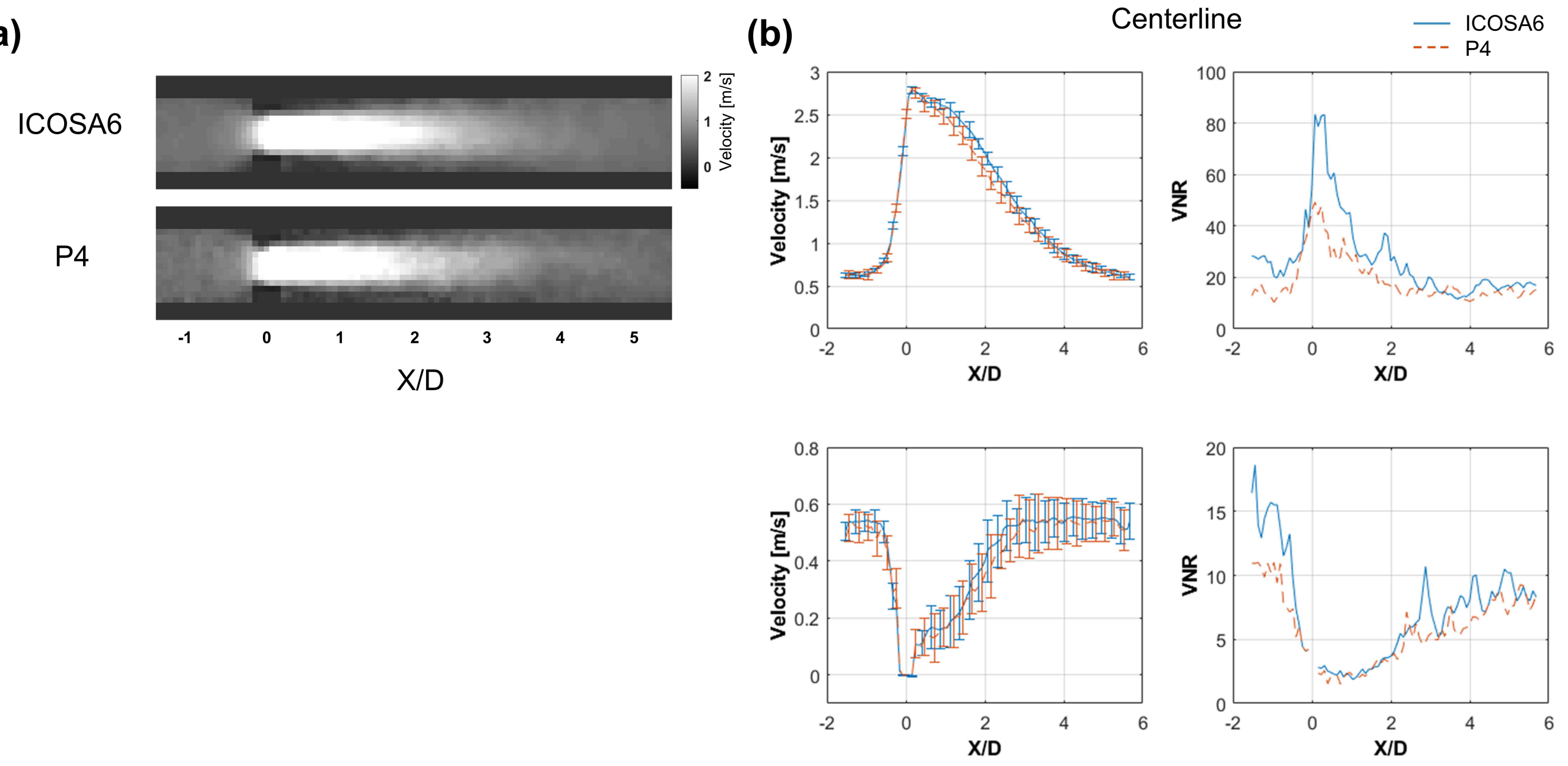

ICOSA6

P4

(a)

X/D

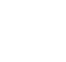

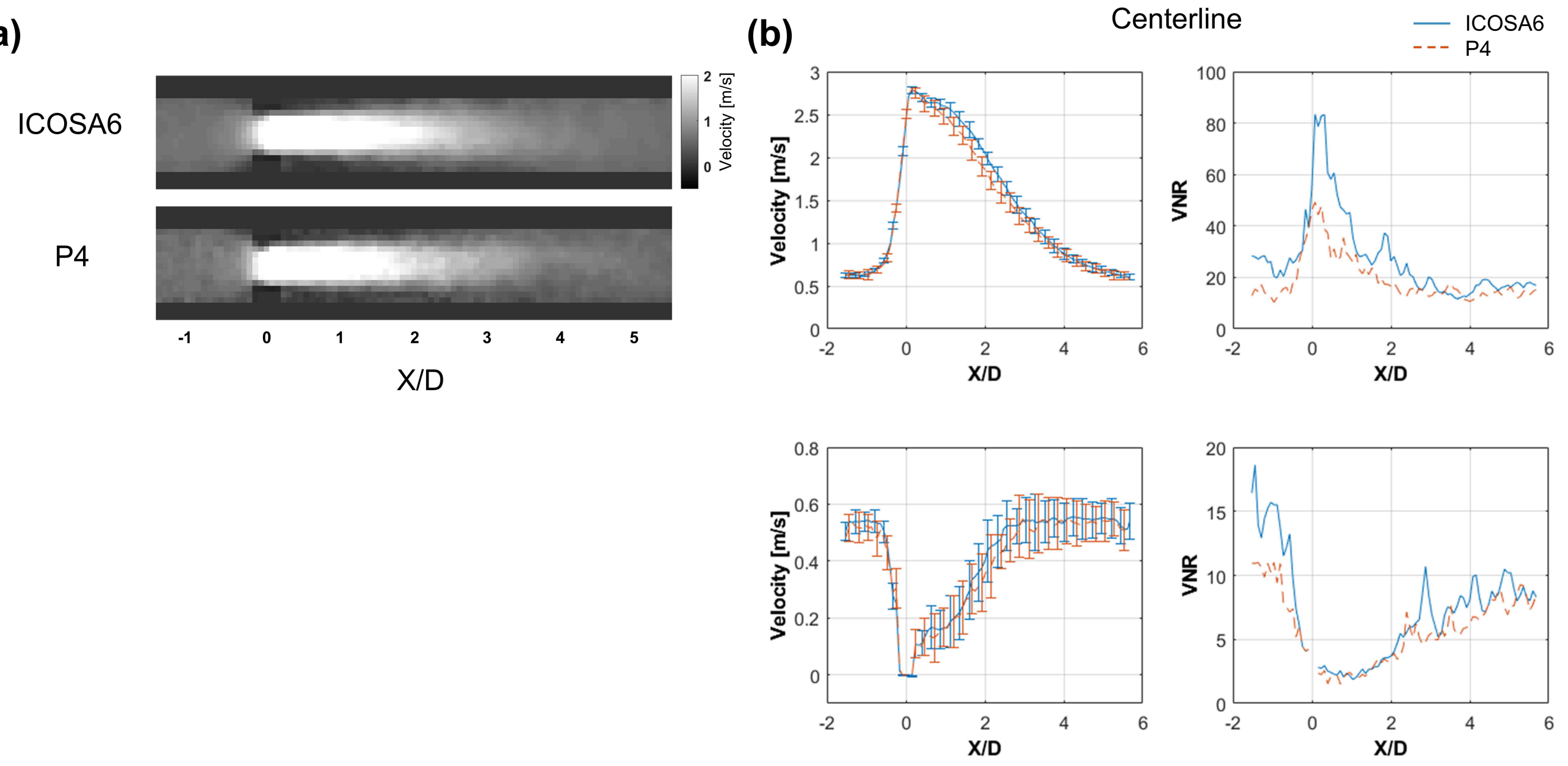

\section{Centerline}
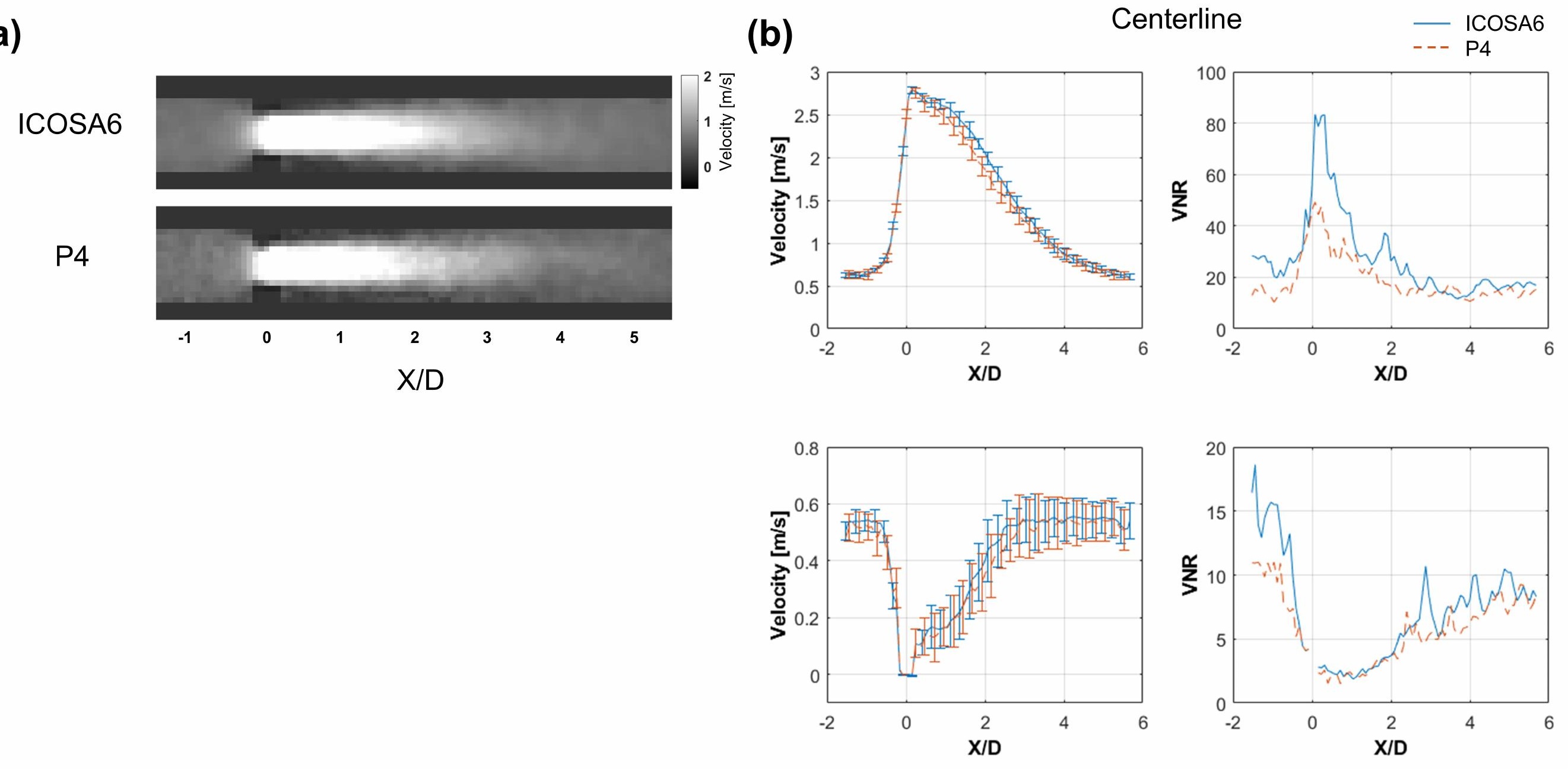
(a)

(a)

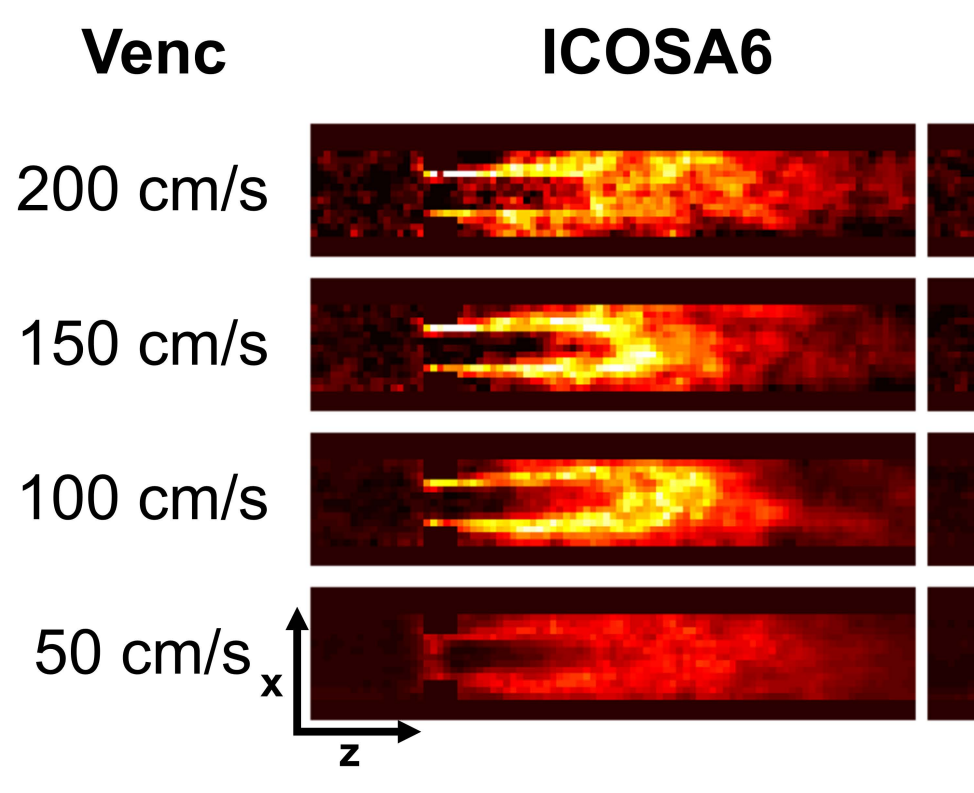

(b)

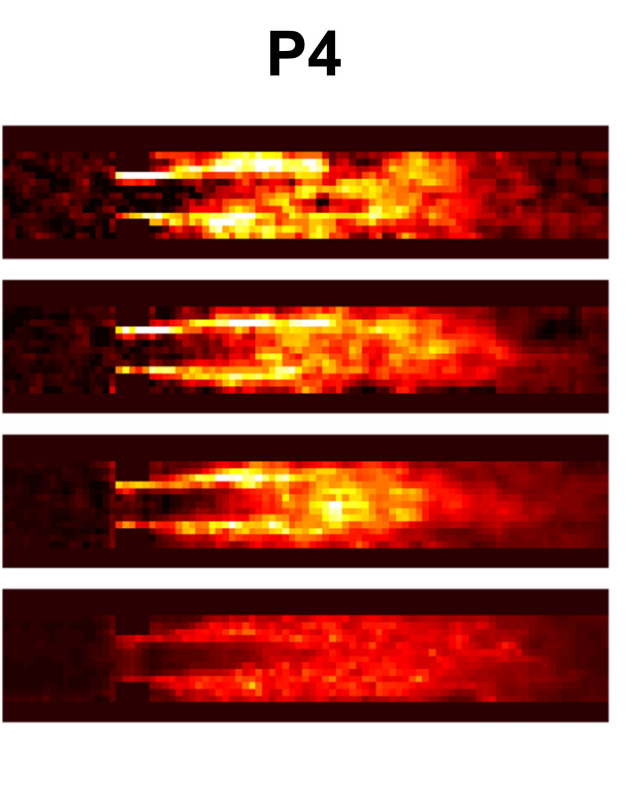

ICOSA6
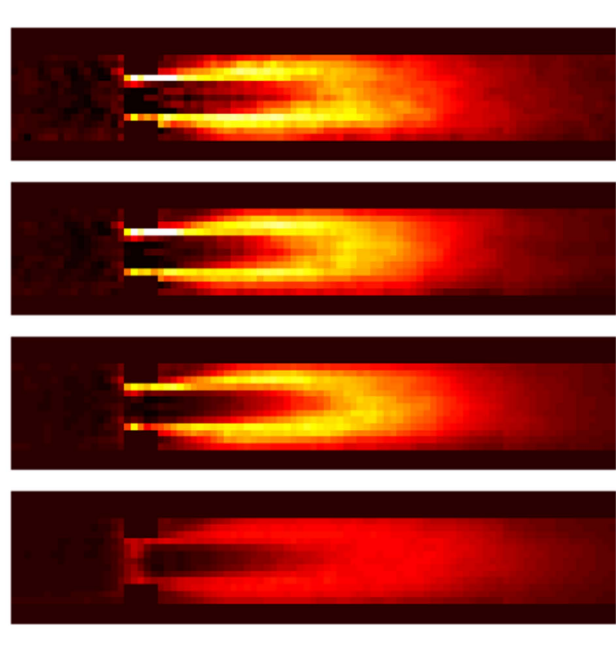

P4

政
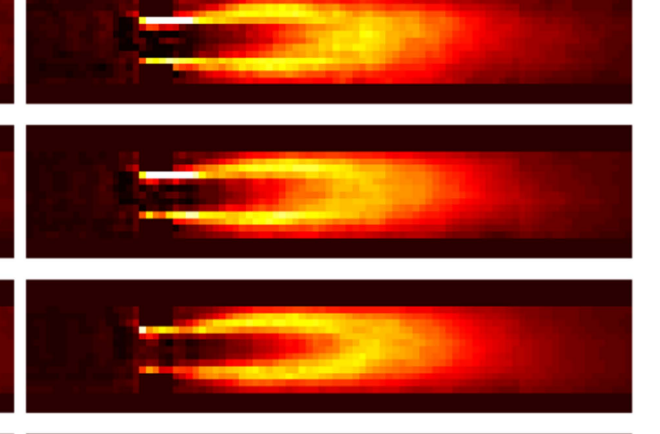

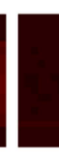

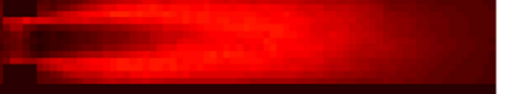

$\begin{array}{lllll}0 & 100 & 200 & 300 & 400\end{array}$

TKE $\left[\mathrm{J} / \mathrm{m}^{3}\right]$ 

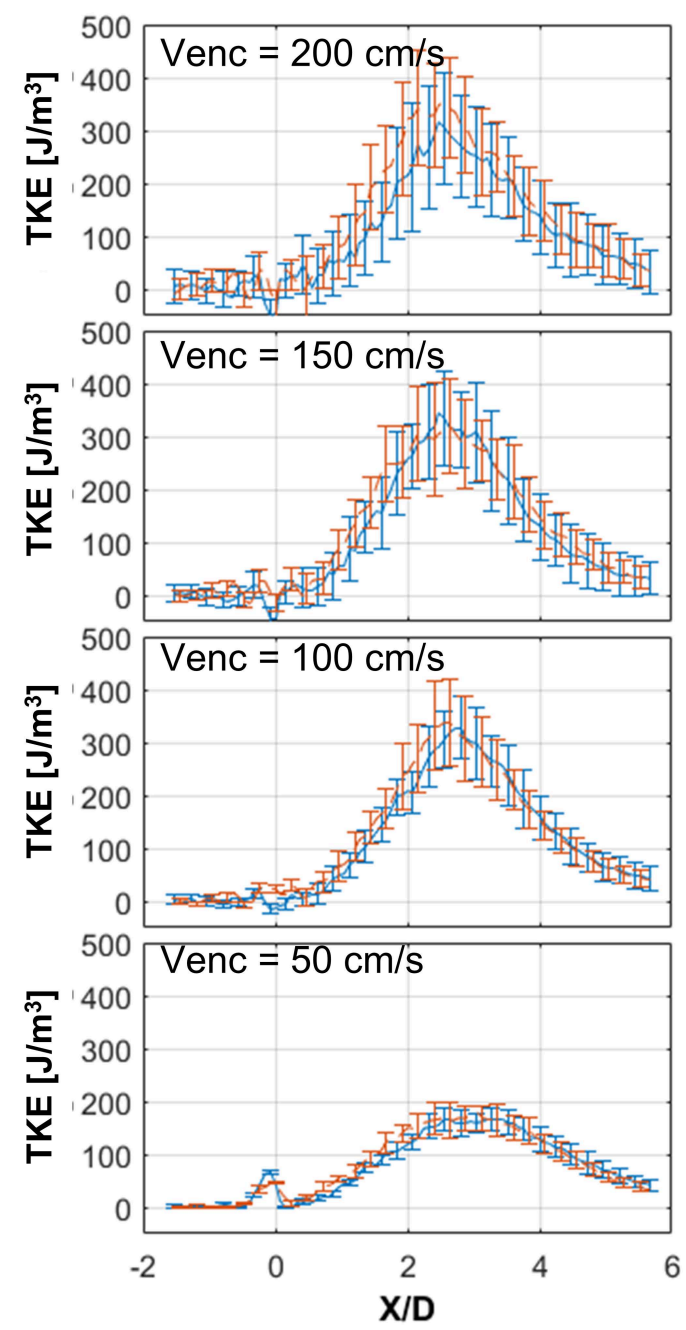

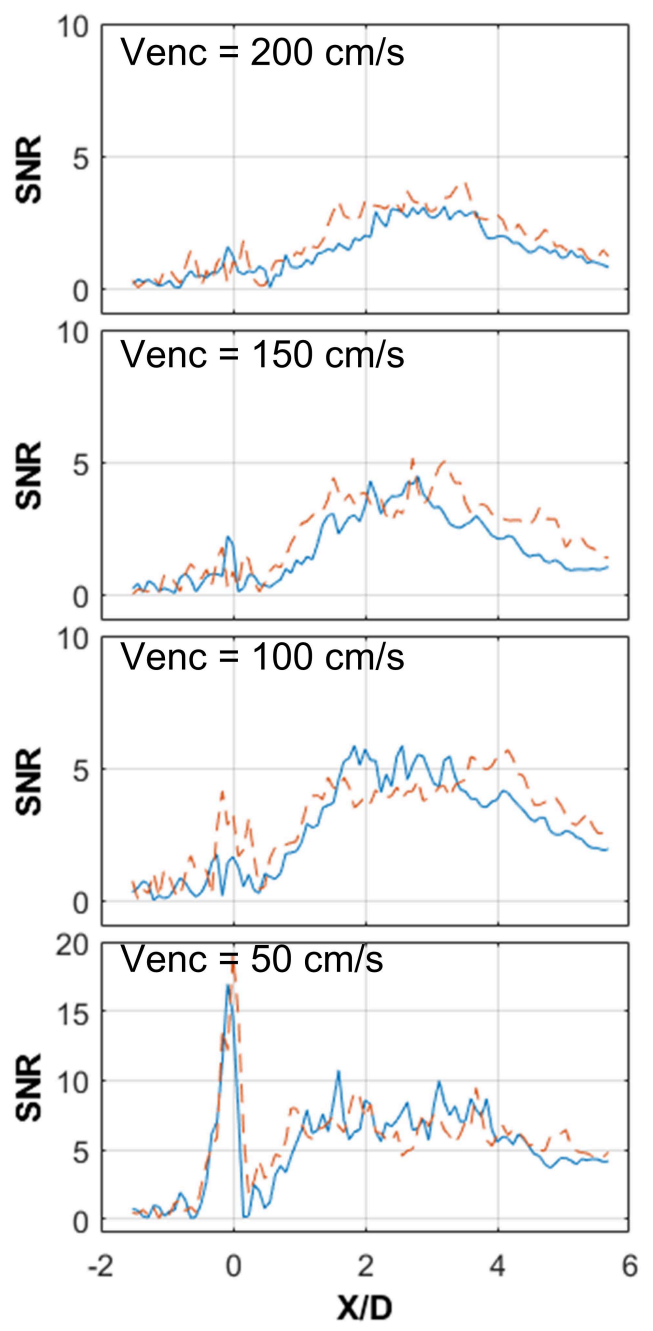




\section{(a)}

With
Contrast agent

Without Contrast agent
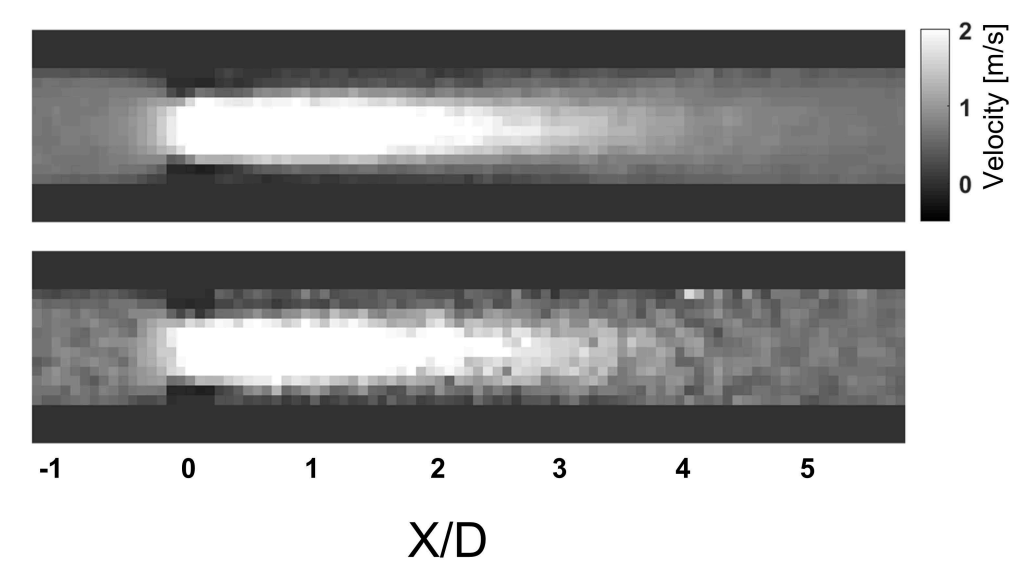

(b)

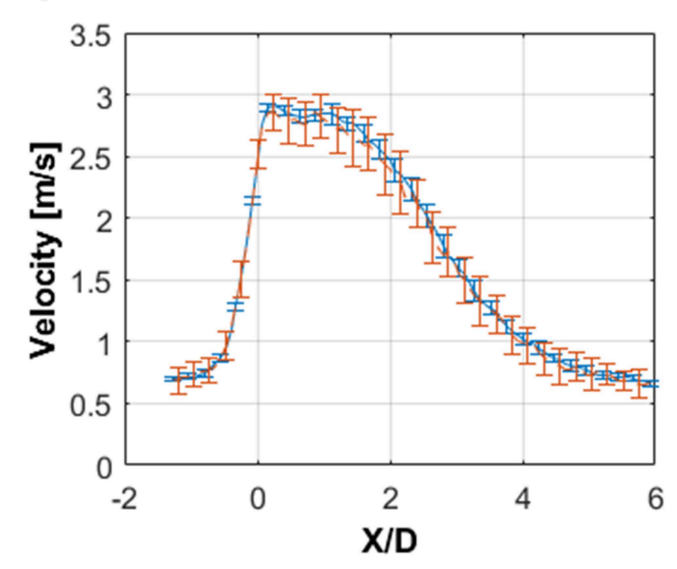

Without Contrast agent

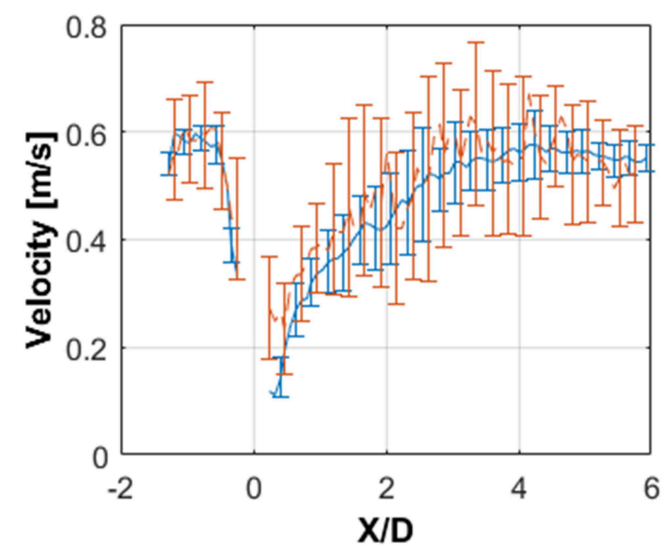

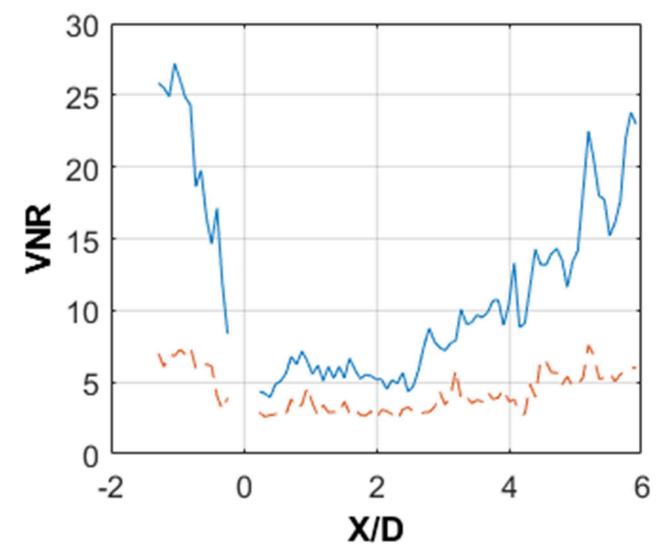


(a)

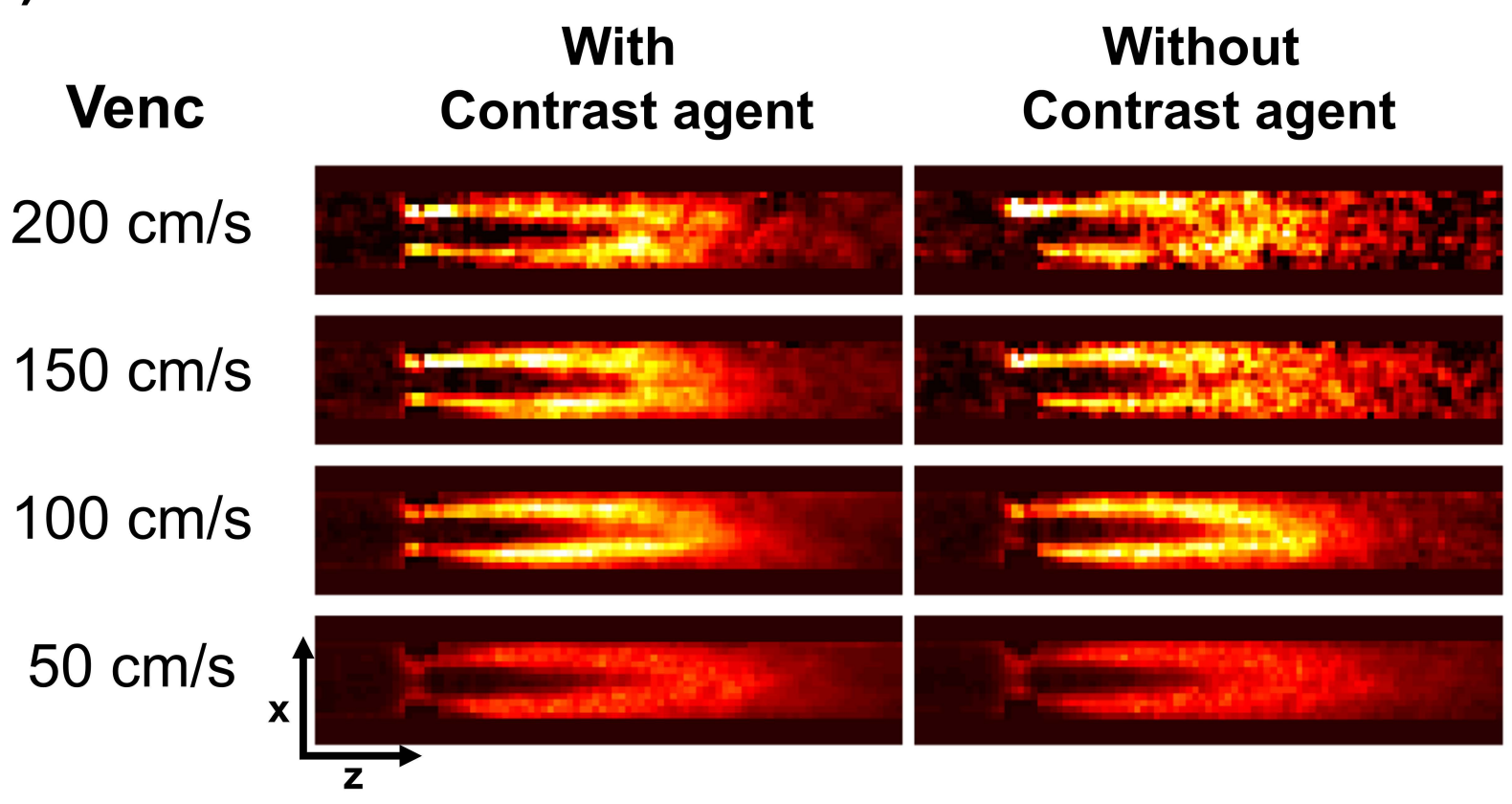

(b)

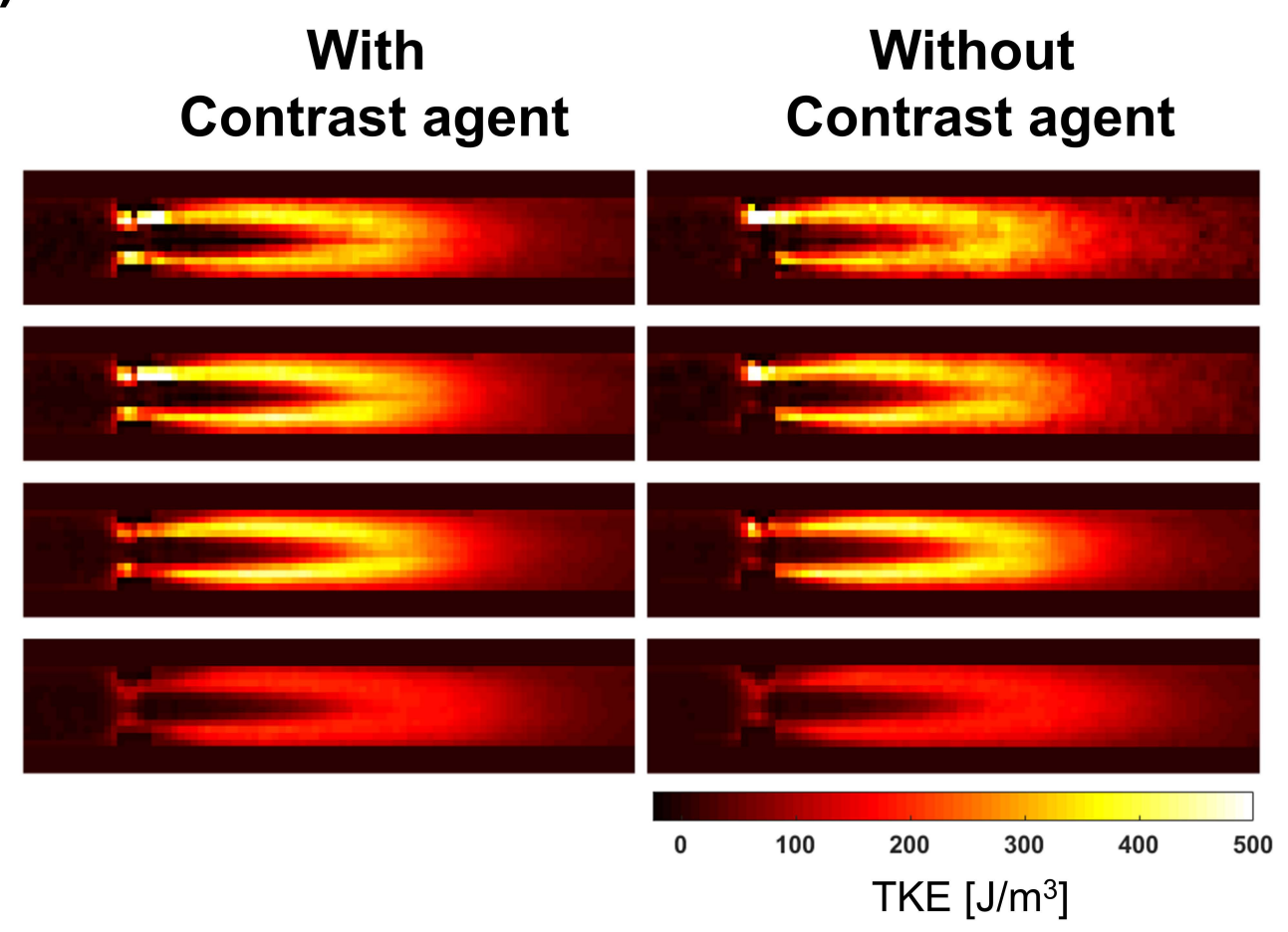



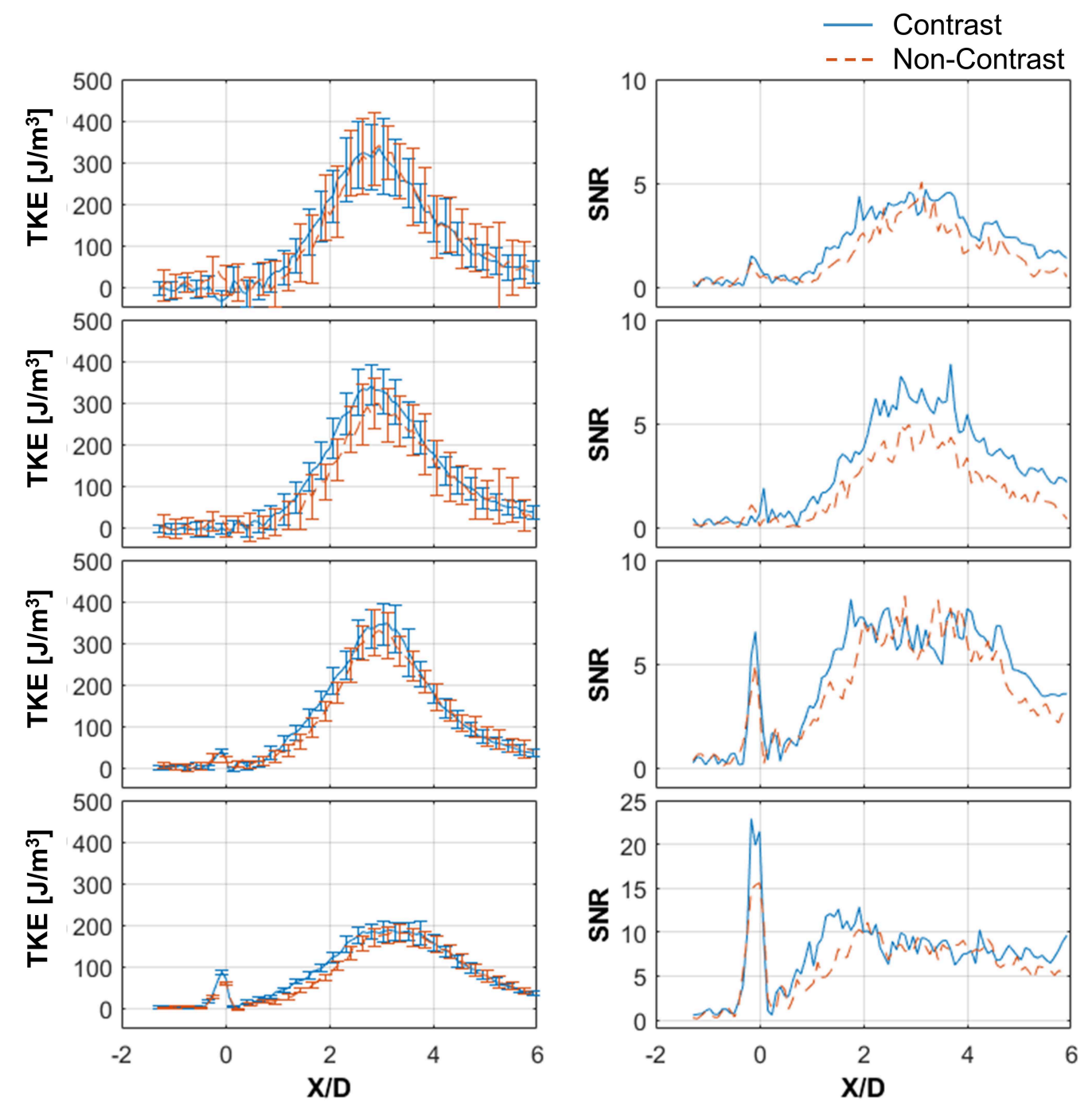
(a)

With

Venc Contrast agent

$200 \mathrm{~cm} / \mathrm{s}$

$150 \mathrm{~cm} / \mathrm{s}$

$100 \mathrm{~cm} / \mathrm{s}$

$50 \mathrm{~cm} / \mathrm{s}$

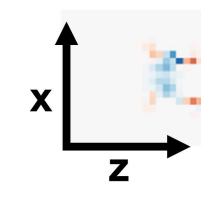

Without

\section{Contrast agent}

mints

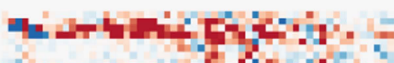

r.

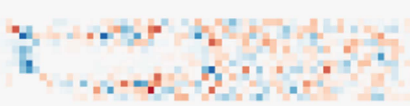

With

Contrast agent

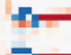

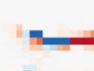

+.
Without

Contrast agent

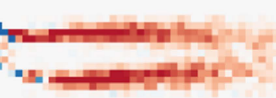

正

$=$

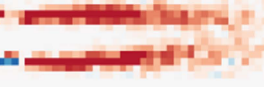

7

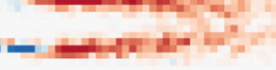




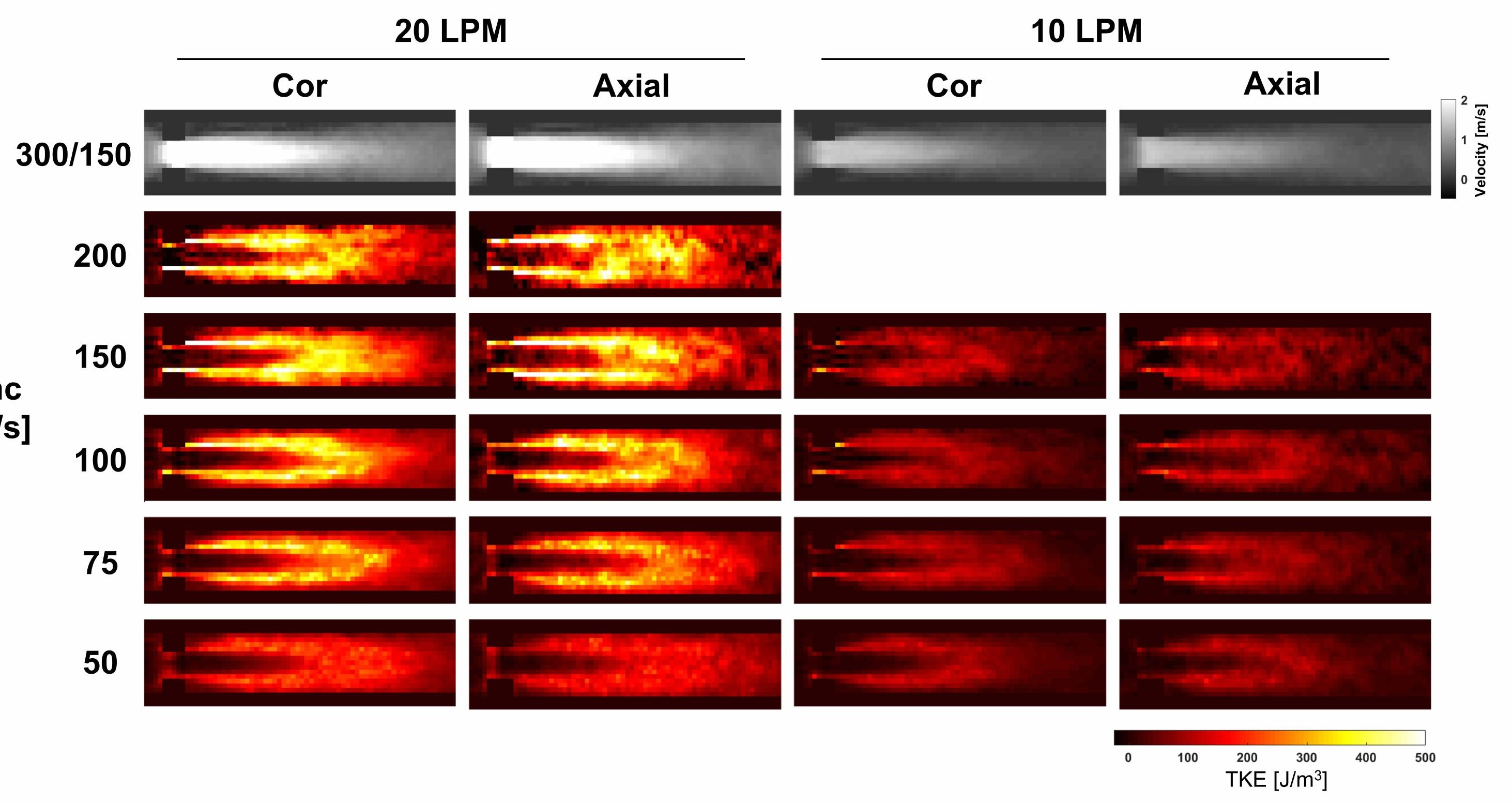




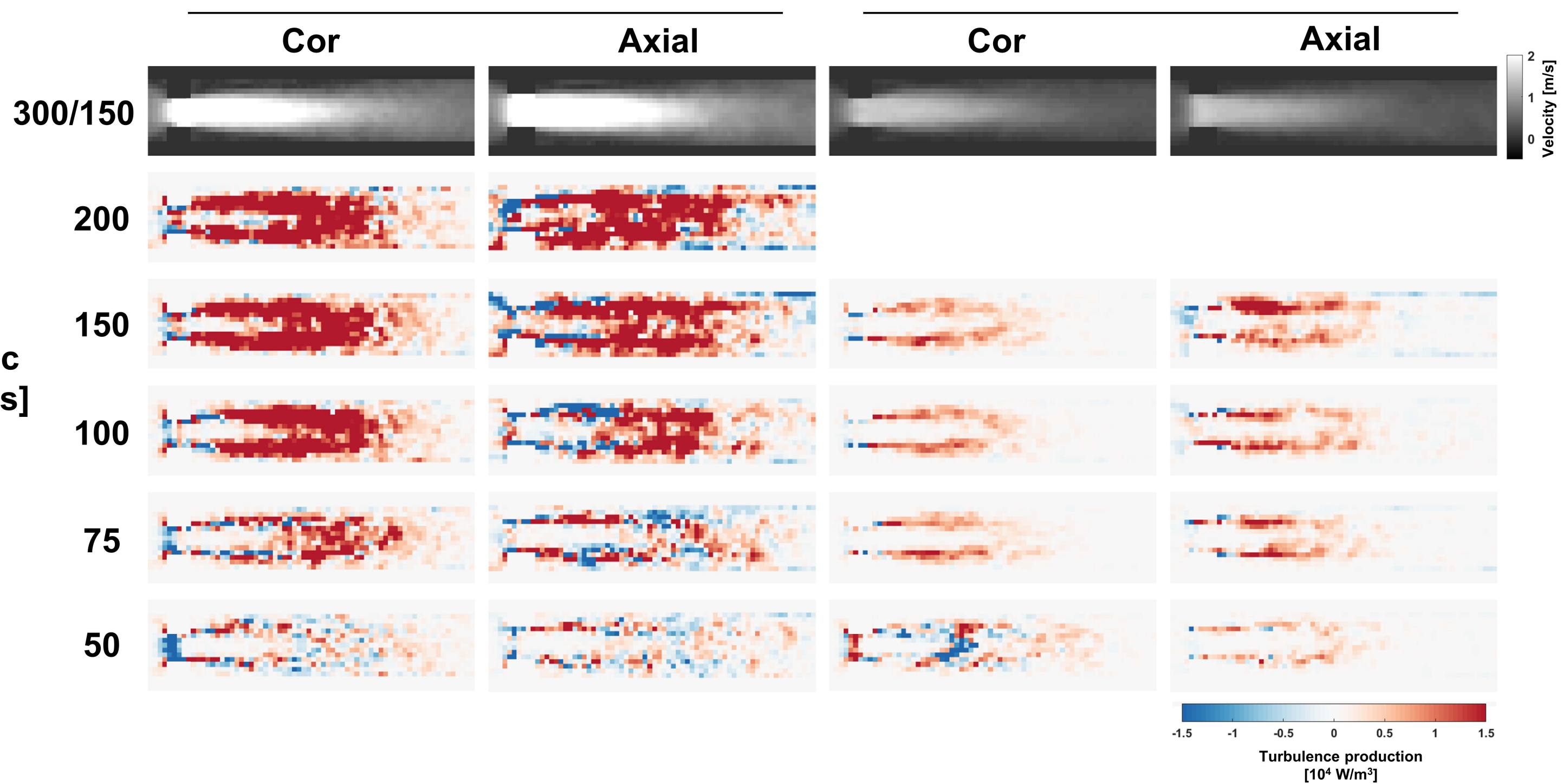




\section{In vitro experiments on ICOSA6 4D flow MRI measurement for the quantification of}

velocity and turbulence parameters

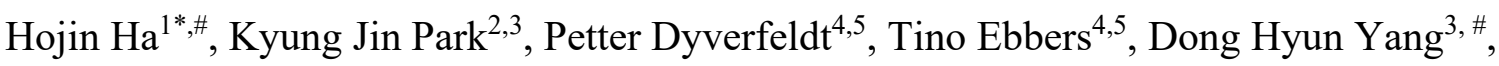

${ }^{1}$ Department of Mechanical and Biomedical Engineering, Kangwon National University, Chuncheon, South Korea.

${ }^{2}$ Department of Electrical and Electronic Engineering, Yonsei Univeristy, Seoul, South Korea.

${ }^{3}$ Department of Radiology, University of Ulsan College of Medicine, Asan Medical Center, Seoul, South Korea.

${ }^{4}$ Department of Health, Medicine and Caring Science, Linköping University, Linköping, Sweden.

${ }^{5}$ Center for Medical Image Science and Visualization (CMIV), Linköping University, Linköping, Sweden.

\# These authors are co-corresponding authors for the study

Corresponding Author: Hojin $\mathrm{Ha}^{*}$

Department of Mechanical and Biomedical Engineering, Kangwon National University, Chuncheon, Republic of Korea.

Phone: $+82-1092871005$

Fax: $+82-33-259-5548$

E-mail: hojinha@kangwon.ac.kr 

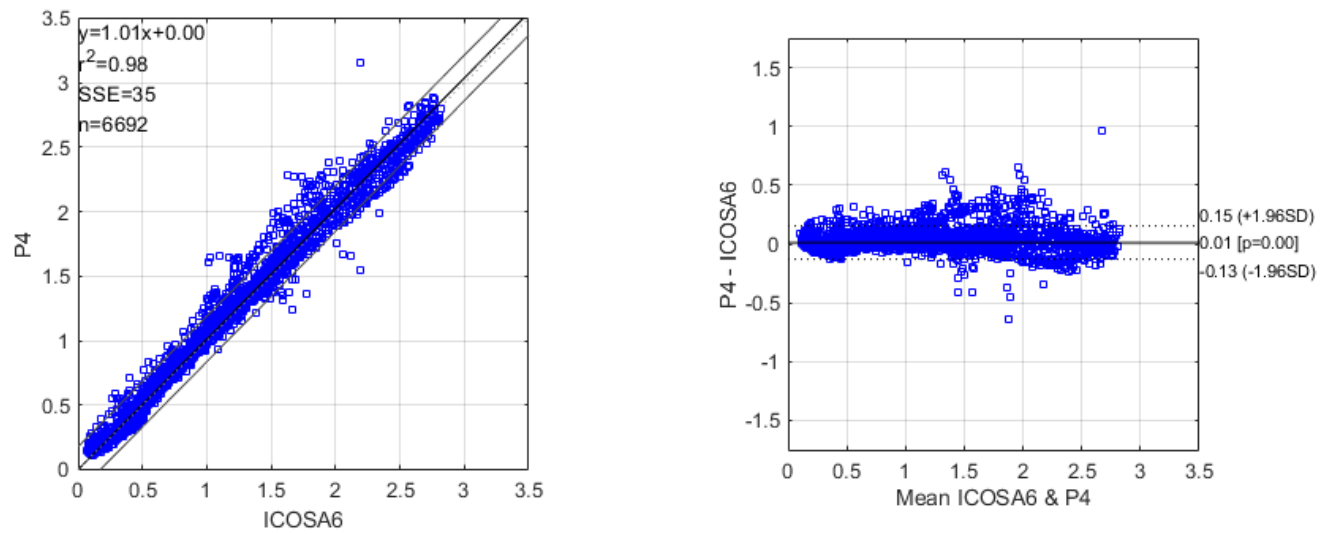

Figure S1. Bland-Altman plot of ICOSA6 and P4 velocity measurements. 


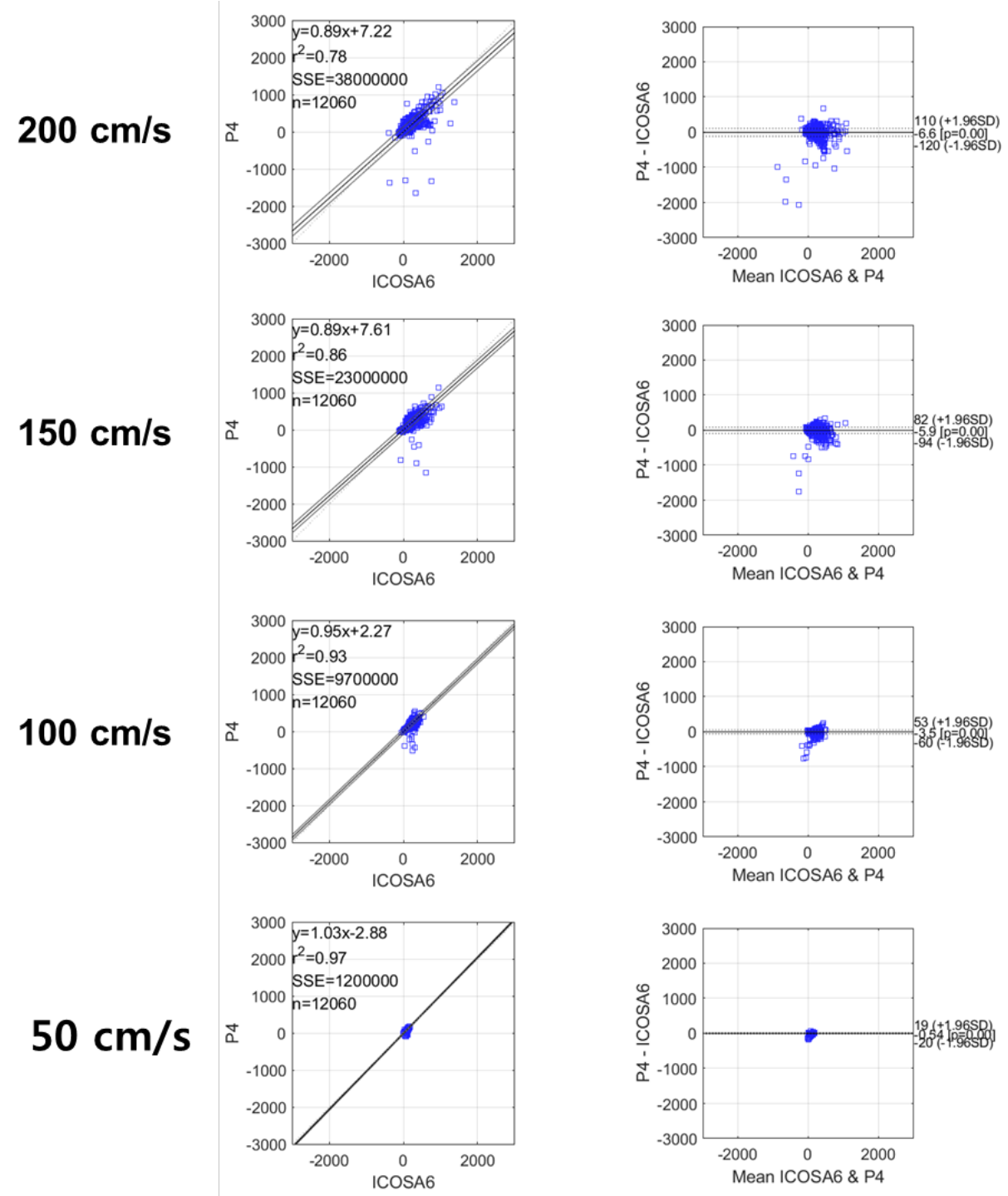

Figure S2. Linear regression and Bland-Altman plots of ICOSA6 and P4 TKE measurements. 
ICOSA6

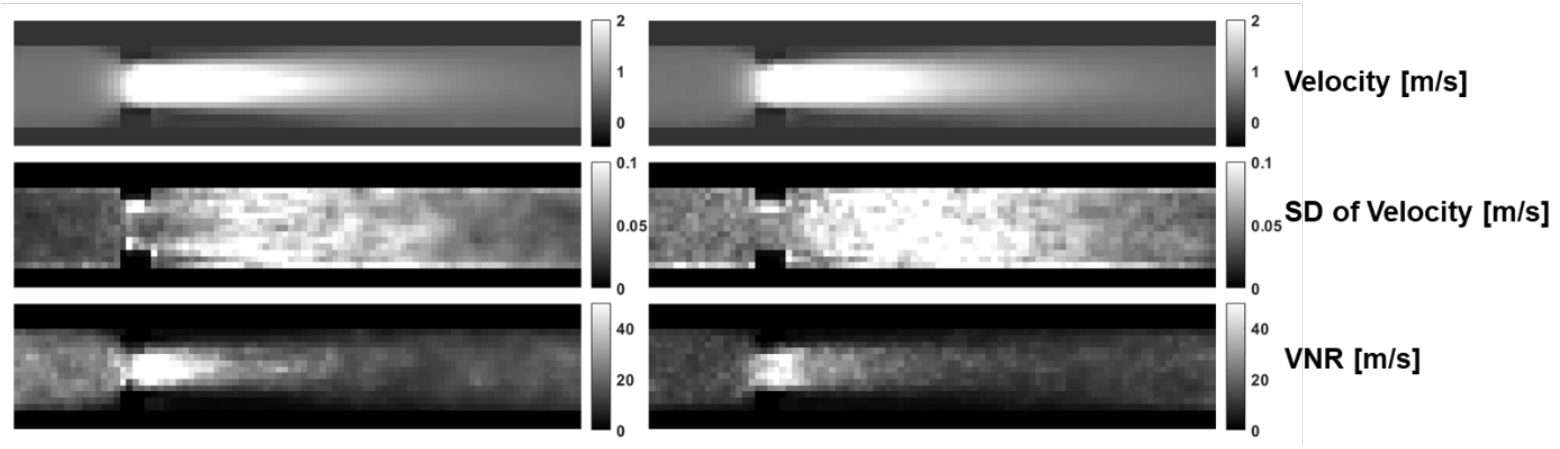

Figure S3. VNR comparison of ICOSA6 and P4 velocity measurements. 
(a)

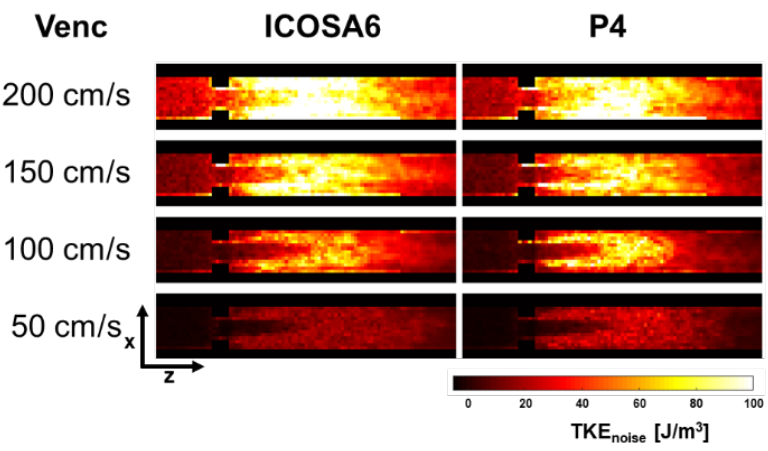

(b)

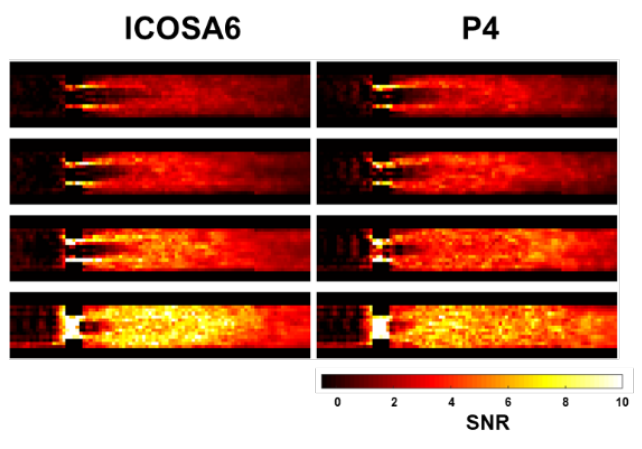

Figure S4. Comparison TKE field measured with ICOSA6 and P4 sequences. (b) TKE noise field, (b) SNR of TKE estimation. 
(a)

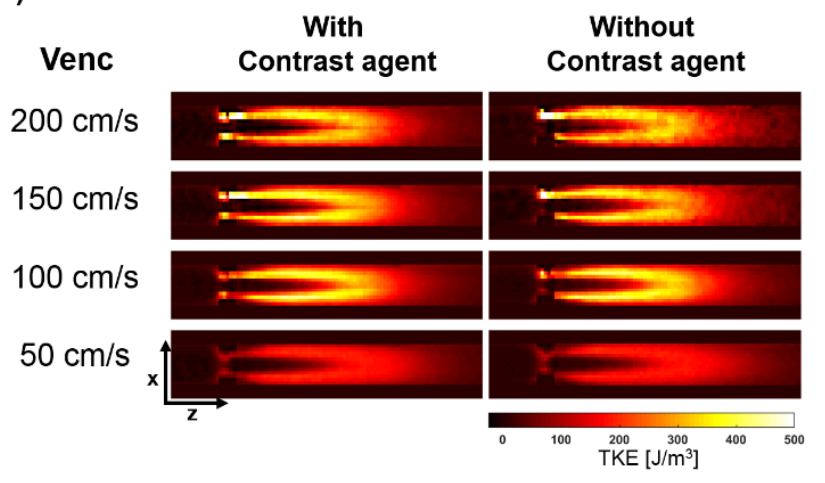

(b)

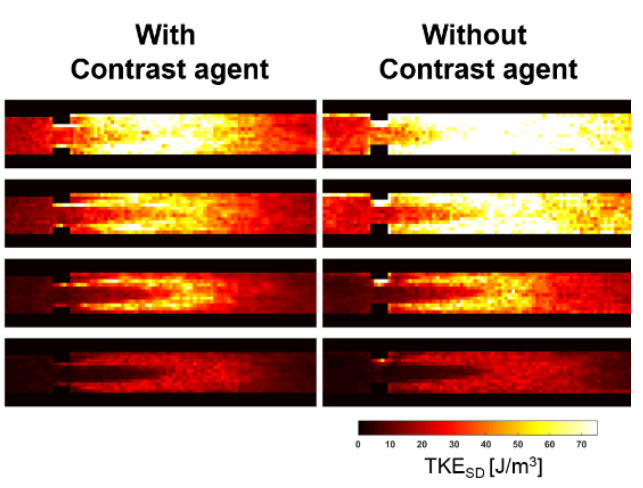

Figure S5. Comparison of ICOSA6 TKE measurements with and without contrast agent. (a) averaged TKE field and (b) standard deviation of the TKE measurements over the temporal phases. 
(a) - ICOSA6

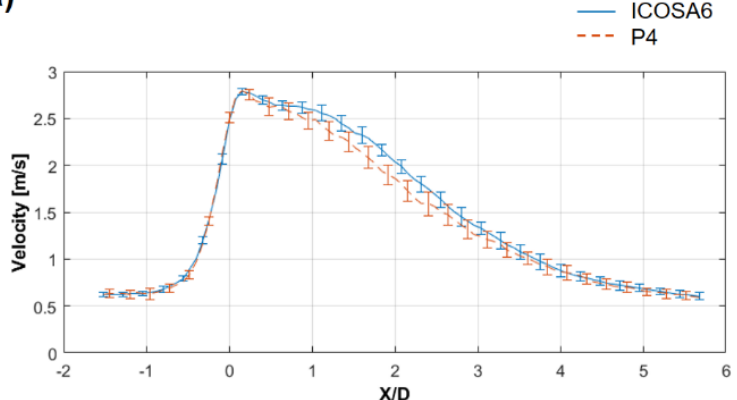

(b)

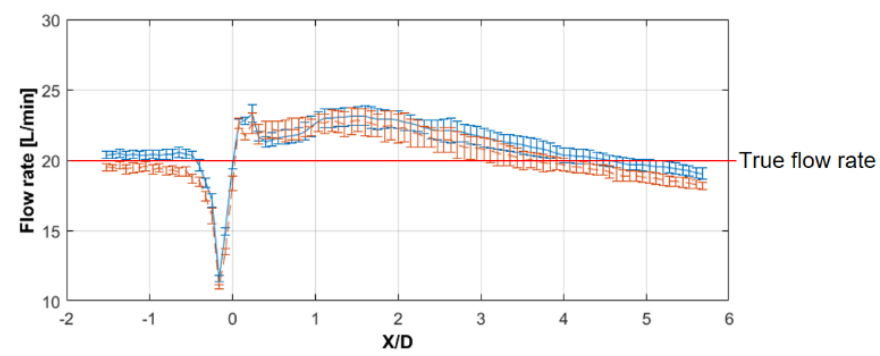

Figure S6. Comparison of ICOSA6 and P4 velocity measurements. (a) velocity field, (b) flow rate along the centerline. $X=0$ at the contraction region and $\mathrm{D}$ is the upstream size of $25 \mathrm{~mm}$. 
Table S1. Comparison of total turbulence production measured with and without contrast agent after removing negative diagonal Reynolds stress elements

\begin{tabular}{cccc}
\hline $\begin{array}{c}\text { Venc } \\
{[\mathbf{c m} / \mathbf{s}]}\end{array}$ & $\begin{array}{c}\text { Total TP } \\
{[\mathbf{m W}]}\end{array}$ & $\begin{array}{c}\text { Total } \mathbf{T P} \mathbf{P}_{\text {Non-Contrast }} \\
{[\mathbf{m W}]}\end{array}$ & Mean Difference [\%] \\
\hline 200 & $537.60 \pm 18.92$ & $493.68 \pm 33.57$ & 8.17 \\
150 & $653.95 \pm 21.23$ & $611.38 \pm 19.16$ & 6.51 \\
100 & $610.30 \pm 15.16$ & $550.89 \pm 18.18$ & 9.73 \\
50 & $70.53 \pm 5.72$ & $53.31 \pm 6.62$ & 24.42 \\
\hline
\end{tabular}

Table S2. Comparison of total turbulence production measured with and without contrast agent after removing negative turbulence production elements

\begin{tabular}{cccc}
\hline $\begin{array}{c}\text { Venc } \\
{[\mathbf{c m} / \mathbf{s}]}\end{array}$ & $\begin{array}{c}\text { Total TP } \\
{[\mathbf{m W}]}\end{array}$ & $\begin{array}{c}\text { Total TPast } \\
{[\mathbf{m W}]}\end{array}$ & Mean Difference [\%] \\
\hline 200 & $1150.69 \pm 16.97$ & $2337.81 \pm 35.43$ & 50.78 \\
150 & $1118.26 \pm 22.55$ & $1970.78 \pm 38.28$ & 43.26 \\
100 & $961.49 \pm 15.98$ & $1569.51 \pm 21.21$ & 38.74 \\
50 & $338.59 \pm 4.74$ & $678.20 \pm 8.41$ & 50.08 \\
\hline
\end{tabular}

Table S3. Summary of peak velocity for all in-vitro experiments

\begin{tabular}{|c|c|c|c|c|c|c|}
\hline Test \# & Objective & $\begin{array}{c}\text { Scan } \\
\text { direction }\end{array}$ & $\begin{array}{c}\text { Flow rate } \\
{[\mathrm{L} / \mathrm{min}]}\end{array}$ & $\begin{array}{l}\text { VENC } \\
{[\mathrm{cm} / \mathrm{s}]}\end{array}$ & Case & $\begin{array}{c}\text { Peak } \\
\text { Velocity } \\
{[\mathrm{m} / \mathrm{s}]} \\
\end{array}$ \\
\hline \multirow{2}{*}{1} & \multirow{2}{*}{$\begin{array}{c}\text { ICOSA6 } \\
\text { vs 4P }\end{array}$} & \multirow{2}{*}{ Coronal } & \multirow{6}{*}{$20 \mathrm{~L} / \mathrm{min}$} & \multirow{2}{*}{450} & ICOSA6 & $2.81 \pm 0.04$ \\
\hline & & & & & $4 P$ & $2.98 \pm 0.05$ \\
\hline \multirow[b]{2}{*}{2} & \multirow{2}{*}{$\begin{array}{l}\text { Contrast } \\
\text { vs Non- } \\
\text { Contrast }\end{array}$} & \multirow[b]{2}{*}{ Coronal } & & \multirow[b]{2}{*}{350} & Contrast & $3.12 \pm 0.03$ \\
\hline & & & & & Non-Contrast & $3.34 \pm 0.06$ \\
\hline \multirow{2}{*}{3} & \multirow{2}{*}{$\begin{array}{l}\text { Coronal } \\
\text { vs Axial }\end{array}$} & \multirow{2}{*}{$\begin{array}{c}\text { Coronal } \\
\text { /Axial }\end{array}$} & & \multirow{2}{*}{300} & Coronal & $2.98 \pm 0.01$ \\
\hline & & & & & Axial & $2.81 \pm 0.04$ \\
\hline \multirow{2}{*}{4} & Coronal & Coronal & \multirow{2}{*}{$10 \mathrm{~L} / \mathrm{min}$} & \multirow{2}{*}{150} & Coronal & $1.56 \pm 0.01$ \\
\hline & vs Axial & /Axial & & & Axial & $1.52 \pm 0.01$ \\
\hline
\end{tabular}

